\title{
Geometric Algebra in Quantum Information Processing
}

\author{
Timothy F. Havel and Chris J. L. Doran
}

\begin{abstract}
This paper develops a geometric model for coupled two-state quantum systems (qubits) using geometric (aka Clifford) algebra. It begins by showing how Euclidean spinors can be interpreted as entities in the geometric algebra of a Euclidean vector space. This algebra is then lifted to Minkowski space-time and its associated geometric algebra, and the insights this provides into how density operators and entanglement behave under Lorentz transformations are discussed. The direct sum of multiple copies of space-time induces a tensor product structure on the associated algebra, in which a suitable quotient is isomorphic to the matrix algebra conventionally used in multi-qubit quantum mechanics. Finally, the utility of geometric algebra in understanding both unitary and nonunitary quantum operations is demonstrated on several examples of interest in quantum information processing.
\end{abstract}

\section{Introduction}

Quantum mechanics attaches physical significance to representations of the rotation group which differ substantially from those studied in classical geometry. Much of the mystery surrounding it is due to this fact. The enormous interest recently generated by proposals to build a quantum computer [Llo95, EJ96, Ste98, WC98, Bro99, BD00] has focussed attention on the simplest possible quantum system: a two-state system or qubit. Our understanding of qubits is based on two distinct geometric models of their states and transformations:

- A complex projective line under the action of $\mathrm{SU}(2)$.

- A Euclidean unit 2-sphere under the action of SO(3).

The first is used almost exclusively in fundamental quantum physics, while the second ("classical") model is used extensively in certain applications, e.g. nuclear magnetic resonance (NMR) spectroscopy [HSTC00]. In particular, in quantum computing a qubit represents a binary 0 or 1 as its state corresponds to one of a pair of conjugate (orthogonal) projective points $|0\rangle$ or $|1\rangle$. These in turn correspond

2000 Mathematics Subject Classification. Primary 81R99; Secondary 15A66, 94 B27.

TH was support during this work by ARO grants DAAD19-01-1-0519 \& DAAD19-01-10678, DAAG55-97-1-0342 from DARPA/MTO, MDA972-01-1-0003 from DARPA/DSO, and by the Cambridge-MIT Institute, Ltd.

CD was supported by an EPSRC Advanced Fellowship. 
to a pair of diametrical points on the unit sphere, which determine the alignment of the qubit with or against the corresponding axis of quantization.

Formally, these two models are related by stereographic projection of the Riemann (unit) sphere onto the Argand plane, the points of which are the ratios of the homogeneous coordinates of points on the projective line (see e.g. [Alt86, FH81]). While this elegant construction describes the mapping between the two representations in a geometric fashion, it does not unite them in a single mathematical structure. This paper provides an informal account of how this is done by geometric (aka Clifford) algebra; in addition, it describes an extension of this formalism to multiqubit systems, and shows that it provides a concise and lucid means of describing the operations of quantum information processing [SCH98, HSTC00]. Significantly, this extension is most naturally derived via the geometric algebra of Minkowski space-time [DLG93], which has also been shown to be an efficient formalism within which to study a very wide range of problems in classical [Hes99, Jan89], relativis-

tic $\left[\right.$ Hes66, Bay96] and fundamental quantum $\left[\mathbf{D L G}^{+} \mathbf{9 6}\right]$ physics. More complete and rigorous accounts may be found in these references, and in [HCST00, Hav01].

\section{Euclidean Geometry and Spinors}

Let $\Re_{3}$ be a three-dimensional Euclidean vector space whose inner product is denoted by $(\boldsymbol{a}, \boldsymbol{b}) \mapsto \boldsymbol{a} \cdot \boldsymbol{b}$. The Clifford or geometric algebra of $\Re_{3}$ is the associative algebra generated by $\mathfrak{R}_{3}$ over $\mathbb{R}$ such that $\boldsymbol{a}^{2}=\|\boldsymbol{a}\|^{2} \equiv \boldsymbol{a} \cdot \boldsymbol{a}$ for all $\boldsymbol{a} \in \mathfrak{R}_{3}$. This algebra will be referred to in the following as the Pauli algebra, and denoted by $\mathcal{G}_{3}$. The interesting thing about this algebra is its geometric interpretation, which will now be described.

To begin, note that every nonzero vector $\boldsymbol{a} \in \mathfrak{R}_{3}$ has an inverse $\boldsymbol{a} /\|\boldsymbol{a}\|^{2}$. In addition, a simple application of the law of cosines shows that the inner product of $\boldsymbol{a}$ with any other vector $\boldsymbol{b} \in \mathfrak{R}_{3}$ is given by the symmetric part of the geometric product:

$$
\begin{aligned}
\frac{1}{2}(\boldsymbol{a} \boldsymbol{b}+\boldsymbol{b} \boldsymbol{a}) & =\frac{1}{2}\left((\boldsymbol{a}+\boldsymbol{b})^{2}-\boldsymbol{a}^{2}-\boldsymbol{b}^{2}\right) \\
& =\frac{1}{2}\left(\|\boldsymbol{a}+\boldsymbol{b}\|^{2}-\|\boldsymbol{a}\|^{2}-\|\boldsymbol{b}\|^{2}\right)=\boldsymbol{a} \cdot \boldsymbol{b}
\end{aligned}
$$

The antisymmetric part, by way of contrast, is called the outer product, and denoted by $(\boldsymbol{a}, \boldsymbol{b}) \mapsto \boldsymbol{a} \wedge \boldsymbol{b} \equiv(\boldsymbol{a} \boldsymbol{b}-\boldsymbol{b} \boldsymbol{a}) / 2$. Since the outer product of two vectors $\boldsymbol{a} \wedge \boldsymbol{b}$ is invariant under inversion in the origin, it cannot itself be a vector. The space $\left\langle\boldsymbol{a} \wedge \boldsymbol{b} \mid \boldsymbol{a}, \boldsymbol{b} \in \Re_{3}\right\rangle$ therefore carries an inequivalent representation of the orthogonal group $\mathrm{O}(3)$, and its elements are accordingly called bivectors. These are most naturally interpreted as oriented plane segments, instead of oriented line segments like vectors in $\mathfrak{R}_{3}$. If we similarly define the outer product of a vector with a bivector and require it to be associative, i.e.

$$
a \wedge(b \wedge c) \equiv \frac{1}{2}(a b c-c b a) \equiv(a \wedge b) \wedge c
$$

$\left(\boldsymbol{a}, \boldsymbol{b}, \boldsymbol{c} \in \mathfrak{R}_{3}\right)$, then it can be shown via straightforward though somewhat lengthy calculations that this product of three vectors is totally antisymmetric, meaning that the outer product generates the well-known exterior algebra $\wedge \mathfrak{R}_{3}$ (cf. [Hes99, Rie58]). The outer product of three vectors is called a trivector, and (since it changes sign under inversion) is most appropriately interpreted as an oriented space segment or volume element. 
The general properties of inner and outer products in the geometric algebras of arbitrary metric vector spaces can be worked out along these lines in a coordinatefree fashion [HS84]. The remainder of this section will focus on how the Pauli algebra is used to describe the quantum mechanics of qubits. In this application it is more common to work with a fixed orthonormal basis $\sigma_{\mathrm{x}}, \sigma_{\mathrm{y}}, \sigma_{\mathrm{z}} \in \mathfrak{R}_{3}$. Quantum mechanics, however, views these basis vectors in a very different way from that taken above, in that they are regarded as operators on a two-dimensional Hilbert space $\mathfrak{H} \approx \mathbb{C}^{2}$ (see e.g. [Sak94]). These operators, in turn, are usually identified with the Pauli matrices

$$
\sigma_{\mathrm{x}} \leftrightarrow \underline{\sigma}_{\mathrm{x}} \equiv\left[\begin{array}{ll}
0 & 1 \\
1 & 0
\end{array}\right], \sigma_{\mathrm{y}} \leftrightarrow \underline{\sigma}_{\mathrm{y}} \equiv\left[\begin{array}{cc}
0 & -\imath \\
\imath & 0
\end{array}\right], \sigma_{\mathrm{z}} \leftrightarrow \underline{\sigma}_{\mathrm{z}} \equiv\left[\begin{array}{cc}
1 & 0 \\
0 & -1
\end{array}\right],
$$

where $\imath$ is an imaginary unit $\left(\imath^{2}=-1\right)$, the underline signifies that the associated symbol is a matrix, and throughout this paper the symbol " $\leftrightarrow$ " should be read as "is represented by" or "is equivalent to". The connection between the two viewpoints lies the fact that these matrices satisfy the defining relations of the abstract Pauli algebra $\mathcal{G}_{3}$, namely

$$
\left(\sigma_{\mu}\right)^{2}=\mathbf{1} \leftrightarrow 1, \quad \boldsymbol{\sigma}_{\mu} \boldsymbol{\sigma}_{\nu}=-\boldsymbol{\sigma}_{\nu} \boldsymbol{\sigma}_{\mu} \quad(\mu, \nu \in\{\mathrm{x}, \mathrm{y}, \mathrm{z}\}, \mu \neq \nu),
$$

and hence constitute a faithful matrix representation of it. This shows, in particular, that $\mathcal{G}_{3}$ is 8 -dimensional as a real linear space. ${ }^{1}$

In most physical situations, these operators (times $\hbar$ ) represent measurements of the intrinsic angular momentum of the qubits, and hence are regarded as generators of rotations in the Lie algebra so(3) over $\mathbb{C}$ satisfying the commutator relation

$$
\frac{1}{2}\left[\sigma_{\mathrm{x}}, \sigma_{\mathrm{y}}\right]=\imath \sigma_{\mathrm{z}},
$$

and its cyclic permutations. In terms of geometric algebra, the left-hand side is just the outer product of the vectors. The right-hand side is somewhat harder to interpret, because the Pauli algebra is defined over the real numbers. The trick is to observe that, in terms of the matrix representation, $\underline{\sigma}_{x} \underline{\sigma}_{y} \underline{\sigma}_{z}=\imath \underline{\mathbf{1}}$. Thus by interpreting the abstract imaginary $\imath$ as the trivector $\iota \equiv \sigma_{\mathrm{x}} \sigma_{\mathrm{y}} \sigma_{\mathrm{z}}=\sigma_{\mathrm{x}} \wedge \sigma_{\mathrm{y}} \wedge \sigma_{\mathrm{z}}$, the angular momentum relations become a triviality:

$$
\sigma_{\mathrm{x}} \wedge \sigma_{\mathrm{y}}=\sigma_{\mathrm{x}} \sigma_{\mathrm{y}}=\sigma_{\mathrm{x}} \sigma_{\mathrm{y}}\left(\sigma_{\mathrm{z}}\right)^{2}=\iota \sigma_{\mathrm{z}}
$$

More generally, the vector cross product is related to the outer product by

$$
\boldsymbol{a} \times \boldsymbol{b}=-\frac{\iota}{2}(\boldsymbol{a b}-\boldsymbol{b a})=-\iota(\boldsymbol{a} \wedge \boldsymbol{b}),
$$

from which it may be seen that multiplication by the unit trivector $\iota$ maps vectors to orthogonal bivectors and vice versa. Since they span a one-dimensional space but change sign under inversion in the origin, trivectors can also be regarded as pseudoscalars. Perhaps the most important thing which geometric algebra contributes to physics are geometric interpretations for the imaginary units which it otherwise uses blindly.

\footnotetext{
${ }^{1}$ In the quantum mechanics literature, the notation $\underline{\boldsymbol{a}} \cdot \underline{\overrightarrow{\boldsymbol{\sigma}}}$ is often used for $\sum_{\mu} a_{\mu} \underline{\boldsymbol{\sigma}}_{\mu}$. Because $\boldsymbol{a}$ is geometrically just a vector in $\mathfrak{R}_{3}$ (not a matrix for it in a basis-dependent representation of $\mathcal{G}_{3}$ ), this is an abuse of the dot-notation for the Euclidean inner product, which is otherwise perhaps the most consistently used notation in all of science. This abuse of notation will not be perpetrated in this paper.
} 
If we denote the induced bivector basis by

$$
\boldsymbol{I} \equiv \sigma_{\mathrm{y}} \wedge \sigma_{\mathrm{z}}, \quad \boldsymbol{J} \equiv \sigma_{\mathrm{z}} \wedge \sigma_{\mathrm{x}}, \quad \boldsymbol{K} \equiv \sigma_{\mathrm{x}} \wedge \sigma_{\mathrm{y}},
$$

it is readily seen that these basis bivectors likewise square to -1 . On multiplying the angular momentum generating relations through by $-1=\iota^{2}$, we obtain

$$
\boldsymbol{J} \boldsymbol{I}=\boldsymbol{K}, \quad \boldsymbol{I} \boldsymbol{K}=\boldsymbol{J}, \quad \boldsymbol{K} \boldsymbol{J}=\boldsymbol{I}, \quad \text { and } \boldsymbol{K} \boldsymbol{J} \boldsymbol{I}=-1 .
$$

This shows that these basis bivectors generate a subalgebra of $\mathcal{G}_{3}$ isomorphic to Hamilton's quaternions [Alt86, Alt89], which is also known as the even subalgebra $\mathcal{G}_{3}^{+}$(since it is generated by the products of even numbers of vectors). It is wellknown that the quaternions' multiplicative group is $\mathbb{R}^{*} \oplus \mathrm{SU}(2)$, which implies that the even subalgebra should be closely related to rotations. This relationship will now be worked out explicitly.

Consider the result of conjugating a vector $\boldsymbol{x}$ by a vector $\boldsymbol{a}$, i.e.

$$
\boldsymbol{a x a ^ { - 1 }}=\boldsymbol{a}\left(\boldsymbol{x}_{\|}+\boldsymbol{x}_{\perp}\right) \boldsymbol{a}^{-1}=\boldsymbol{a a ^ { - 1 }} \boldsymbol{x}_{\|}-\boldsymbol{a a ^ { - 1 }} \boldsymbol{x}_{\perp},
$$

where we have split $\boldsymbol{x}=\left(\boldsymbol{x} \cdot \boldsymbol{a}^{-1}+\boldsymbol{x} \wedge \boldsymbol{a}^{-1}\right) \boldsymbol{a} \equiv \boldsymbol{x}_{\|}+\boldsymbol{x}_{\perp}$ into its parts parallel and perpendicular to $\boldsymbol{a}$. This shows that $-\boldsymbol{a x} \boldsymbol{a}^{-1}$ is the reflection of $\boldsymbol{x}$ in the plane orthogonal to $\boldsymbol{a}$. ¿From the well-known fact that the composition of two reflections is a rotation by twice the lessor angle between their planes and about these planes' line of intersection, it follows that conjugating a vector by an element of the even subalgebra just rotates it accordingly:

$$
(\boldsymbol{b a}) \boldsymbol{x}(\boldsymbol{b a})^{-1}=\boldsymbol{b a} \boldsymbol{x} \boldsymbol{a}^{-1} \boldsymbol{b}^{-1}=\frac{\boldsymbol{b a} \boldsymbol{x} \boldsymbol{a b}}{\|\boldsymbol{a}\|^{2}\|\boldsymbol{b}\|^{2}}
$$

Let $\boldsymbol{u} \equiv \boldsymbol{a} /\|\boldsymbol{a}\|, \boldsymbol{v} \equiv \boldsymbol{b} /\|\boldsymbol{b}\|$ and $\boldsymbol{R} \equiv \boldsymbol{v} \boldsymbol{u}$ be the corresponding unit quaternion. Then $\boldsymbol{R}=\cos (\theta / 2)-\boldsymbol{\iota r} \sin (\theta / 2)$ where $\cos (\theta / 2)=\boldsymbol{u} \cdot \boldsymbol{v}$ and $\boldsymbol{\iota} \boldsymbol{r} \equiv \boldsymbol{u} \wedge \boldsymbol{v} /\|\boldsymbol{u} \wedge \boldsymbol{v}\|$. Moreover, the inverse $(\boldsymbol{v} \boldsymbol{u})^{-1}$ is now simply the reverse $\boldsymbol{u} \boldsymbol{v} \equiv(\boldsymbol{v} \boldsymbol{u})^{\dagger}$, which in turn corresponds to the conjugate quaternion $\boldsymbol{R}^{\dagger} \equiv \cos (\theta / 2)+\boldsymbol{\iota r} \sin (\theta / 2)$. This reversal operation on $\mathcal{G}_{3}^{+}$extends to a well-defined anti-automorphism of $\mathcal{G}_{3}$, which corresponds to Hermitian conjugation in its representation by Pauli matrices. On splitting $\boldsymbol{x}$ into its parts parallel $\boldsymbol{x}_{\|}$and perpendicular $\boldsymbol{x}_{\perp}$ to $\boldsymbol{r}$ as above, the rotation may now be written as $\boldsymbol{R} \boldsymbol{x} \boldsymbol{R}^{\dagger}=\boldsymbol{R}\left(\boldsymbol{x}_{\|}+\boldsymbol{x}_{\perp}\right) \boldsymbol{R}^{\dagger}=\boldsymbol{x}_{\|}+\boldsymbol{x}_{\perp}\left(\boldsymbol{R}^{\dagger}\right)^{2}$

$$
\begin{aligned}
& =\boldsymbol{x}_{\|}+\boldsymbol{x}_{\perp}\left(\cos ^{2}(\theta / 2)-\sin ^{2}(\theta / 2)+2 \iota \boldsymbol{r} \cos (\theta / 2) \sin (\theta / 2)\right) \\
& =\boldsymbol{x}_{\|}+\boldsymbol{x}_{\perp}(\cos (\theta)+\iota \boldsymbol{r} \sin (\theta))
\end{aligned}
$$

and so may be viewed as multiplication of $\boldsymbol{x}_{\perp}$ by the "complex number" $\cos (\theta)+$ $\iota \boldsymbol{r} \sin (\theta)$ in the Argand plane defined by the bivector $\iota \boldsymbol{r}$.

By collecting even and odd powers in its Taylor series, it may be seen that any unit quaternion can be written as the exponential of a bivector orthogonal to the axis of rotation $\boldsymbol{r}$ :

$$
e^{-\iota \boldsymbol{r} \theta / 2}=1-\iota \boldsymbol{r} \frac{\theta}{2}-\frac{1}{2}\left(\frac{\theta}{2}\right)^{2}+\cdots=\cos (\theta / 2)-\iota \boldsymbol{r} \sin (\theta / 2)
$$

This is formally analogous to a complex exponential, and is also in accord with our previous observation that the space of bivectors is isomorphic to the Lie algebra su $(2) \approx \operatorname{so}(3)$ under the commutator product. The pair $[\cos (\theta / 2) ; \sin (\theta / 2) \boldsymbol{r}]$ are known as Euler-Rodrigues parameters for the rotation group $\mathrm{SO}(3)$; since 
$[-\cos (\theta / 2) ;-\sin (\theta / 2) \boldsymbol{r}]$ determines the same rotation, this parametrization is twoto-one. A one-to-one parametrization is obtained from the outer exponential, i.e.

$$
\wedge^{-\iota \boldsymbol{r} \tau}=1-\boldsymbol{\iota r} \tau-\frac{1}{2} \boldsymbol{r} \wedge \boldsymbol{r} \tau^{2}+\cdots=1-\boldsymbol{\iota r} \tau \quad(\text { since } \boldsymbol{r} \wedge \boldsymbol{r}=0) .
$$

The squared norm of this outer exponential is $1+\tau^{2}$, so that the normalized outer exponential equals the usual exponential if we set $\tau=\tan (\theta / 2)$. Because $\boldsymbol{t} \equiv$ $\tan (\theta / 2) \boldsymbol{r}$ is the four-dimensional stereographic projection of $[\cos (\theta) ; \sin (\theta) \boldsymbol{r}]$ from $\theta=\pi$, it has been called the stereographic parameter for $\mathrm{SO}(3)$. Note, however, that this parametrization does not include rotations by $\pi$.

Another two-to-one parametrization of rotations is given by the Cayley-Klein parameters $\left[\psi_{1} ; \psi_{2}\right] \in \mathbb{C}^{2}$, where

$$
\begin{aligned}
& \psi_{1} \equiv \cos (\theta / 2)-\imath \sin (\theta / 2)\left(\boldsymbol{r} \cdot \boldsymbol{\sigma}_{\mathbf{z}}\right), \\
& \psi_{2} \equiv \sin (\theta / 2)\left(\boldsymbol{r} \cdot \boldsymbol{\sigma}_{\mathrm{x}}\right)+\imath \sin (\theta / 2)\left(\boldsymbol{r} \cdot \boldsymbol{\sigma}_{\mathrm{y}}\right) .
\end{aligned}
$$

The corresponding SU(2) matrix is simply

$$
\underline{\boldsymbol{\Psi}}=\left[\begin{array}{cc}
\psi_{1} & -\psi_{2}^{*} \\
\psi_{2} & \psi_{1}^{*}
\end{array}\right] .
$$

It follows that the complex column vector $|\psi\rangle \equiv\left[\psi_{1} ; \psi_{2}\right]$ itself transforms under left-multiplication with matrices in $\mathrm{SU}(2)$, which is commonly described in quantum mechanics by calling it a spinor. In particular, the spinors $|0\rangle \equiv[1 ; 0]$ and $|1\rangle \equiv$ $[0 ; 1]$ are those commonly used in quantum computing to store binary information. Since the Cayley-Klein parameters uniquely determine the $\mathrm{SU}(2)$ matrix, however, we can just as well regard spinors as entities in SU(2), e.g. $|0\rangle \leftrightarrow \underline{\mathbf{1}}$ and $|1\rangle \leftrightarrow-\imath \underline{\boldsymbol{\sigma}}_{\mathrm{y}}$. The usual action of SU(2) on spinors then becomes the left-regular action of $\mathrm{SU}(2)$ on itself.

The representation of $\mathrm{SU}(2)$ used above depends upon the choice of coordinate system: Changing to a different the coordinate system gives a different (though equivalent) representation. Recalling that $\mathrm{SU}(2)$ is isomorphic to the multiplicative group of unit elements (quaternions) in the even subalgebra $\mathcal{G}_{3}^{+}$, a coordinate-free or geometric interpretation of spinors is obtained by regarding them as elements of $\mathcal{G}_{3}^{+}$ itself. This interpretation of spinors as entities in ordinary Euclidean geometry was first pointed out by Hestenes over thirty years ago [Hes66], but physicists persist in putting operators and operands into separate spaces, and in working with a matrix representation instead of directly with the geometric entities themselves. The perceived nonintuitive nature of quantum mechanics is due in large part to the resulting confusion over the geometric meaning of the objects with which it deals, which is spelled out explicitly in geometric algebra.

As another example, consider how the density operator of an "ensemble" of qubits can be interpreted in geometric algebra. This operator $\boldsymbol{\rho}$ is usually defined via a matrix representation as $\boldsymbol{\rho} \equiv \overline{|\psi\rangle\langle\psi|}$, where the overline denotes the average over the ensemble. As first observed by von Neumann, this matrix contains all the information needed to compute the ensemble average expectation values of the qubit observables, since

$$
\overline{\left\langle\psi\left|\underline{\boldsymbol{\sigma}}_{\mu}\right| \psi\right\rangle}=\overline{\operatorname{tr}\left(\underline{\boldsymbol{\sigma}}_{\mu}|\psi\rangle\langle\psi|\right)}=\operatorname{tr}\left(\underline{\boldsymbol{\sigma}}_{\mu} \overline{|\psi\rangle\langle\psi|}\right)=\operatorname{tr}\left(\underline{\boldsymbol{\sigma}}_{\mu} \underline{\boldsymbol{\rho}}\right)
$$

$(\mu \in\{\mathrm{x}, \mathrm{y}, \mathrm{z}\})$. To translate this into geometric algebra, we set the second column of $\underline{\boldsymbol{\Psi}}$ to zero by right-multiplying it by the idempotent matrix $\underline{\boldsymbol{E}}_{+} \equiv\left(\underline{\mathbf{1}}+\underline{\boldsymbol{\sigma}}_{\mathrm{z}}\right) / 2$, 
i.e.

$$
\underline{\boldsymbol{\Psi}}_{+} \underline{\boldsymbol{E}}_{+} \equiv\left[\begin{array}{cc}
\psi_{1} & -\psi_{2}^{*} \\
\psi_{2} & \psi_{1}^{*}
\end{array}\right]\left[\begin{array}{cc}
1 & 0 \\
0 & 0
\end{array}\right]=\left[\begin{array}{ll}
\psi_{1} & 0 \\
\psi_{2} & 0
\end{array}\right] .
$$

This corresponds to projecting $\boldsymbol{\Psi} \in \mathcal{G}_{3}^{+}$onto a left-ideal in $\mathcal{G}_{3}$, and allows the dyadic product $|\psi\rangle\langle\psi|$ in Eq. (2.17) to be written as:

$$
|\psi\rangle\langle\psi|=\left[\begin{array}{cc}
\psi_{1} & 0 \\
\psi_{2} & 0
\end{array}\right]\left[\begin{array}{cc}
\psi_{1}^{*} & \psi_{2}^{*} \\
0 & 0
\end{array}\right] \equiv\left(\underline{\boldsymbol{\Psi}}_{\boldsymbol{E}}\right)\left(\underline{\boldsymbol{\Psi}}_{\boldsymbol{E}} \underline{\underline{E}}_{+}^{\dagger}\right.
$$

Thus the interpretation of the density operator in geometric algebra is

$$
\boldsymbol{\rho}=\overline{\left(\boldsymbol{\Psi} \boldsymbol{E}_{+}\right)\left(\boldsymbol{\Psi} \boldsymbol{E}_{+}\right)^{\dagger}}=\overline{\boldsymbol{\Psi} \boldsymbol{E}_{+} \boldsymbol{\Psi}^{\dagger}}=\frac{1}{2}\left(1+\overline{\boldsymbol{\Psi} \boldsymbol{\sigma}_{\mathrm{z}} \boldsymbol{\Psi}^{\dagger}}\right)
$$

(cf. [SLD99]). The vector part $\boldsymbol{p} \equiv\langle\boldsymbol{\rho}\rangle_{1}=\overline{\boldsymbol{\Psi} \boldsymbol{\sigma}_{\mathrm{z}} \boldsymbol{\Psi}^{\dagger}}$ is called the polarization vector (in optics, its components are known as the Stokes parameters [BBDH93], while in NMR it is known as the Bloch vector after the pioneer of NMR who rediscovered it [Blo46]). Its length is $\|\boldsymbol{p}\| \leq 1$ with equality if and only if all members of the ensemble are in the same state $\boldsymbol{\Psi}$. In this case the ensemble is said to be in a pure state, and the density operator is itself an idempotent $(1+\boldsymbol{p}) / 2$, where $\boldsymbol{p} \equiv \boldsymbol{\Psi} \boldsymbol{\sigma}_{\mathbf{z}} \boldsymbol{\Psi}^{\dagger}$. For an ensemble in a general mixed state, the length of the ensemble-average polarization vector measures the degree of alignment among the (unit length) polarization vectors of the individual members of the ensemble, and is called the polarization of the ensemble.

In many physical situations there is a natural reference direction; for example, in NMR computing the qubits are spin $1 / 2$ atomic nuclei whose intrinsic magnetic dipoles have been polarized by the application of a strong magnetic field [HCST00]. ¿From a geometric perspective, however, the density operator is just the sum of a scalar and a vector, which for a pure state is related to the corresponding "spinor" by rotation of a fixed reference vector (conventionally taken to be $\sigma_{\mathbf{z}}$ as above) by $\boldsymbol{\Psi}$. Since the trace in the standard matrix representation is simply twice the scalar part $\left\langle_{-}\right\rangle_{0}$ of the corresponding expression in geometric algebra, the ensembleaverage expectation value

$$
\frac{1}{2} \operatorname{tr}\left(\underline{\boldsymbol{\sigma}}_{\mu} \underline{\boldsymbol{\rho}}\right) \leftrightarrow\left\langle\boldsymbol{\sigma}_{\mu} \boldsymbol{\rho}\right\rangle_{0}=\left\langle\boldsymbol{\sigma}_{\mu} \boldsymbol{\Psi} \boldsymbol{\sigma}_{\mathrm{z}} \boldsymbol{\Psi}^{\dagger}\right\rangle_{0}=\boldsymbol{\sigma}_{\mu} \cdot\left(\boldsymbol{\Psi} \boldsymbol{\sigma}_{\mathrm{z}} \boldsymbol{\Psi}^{\dagger}\right)=\boldsymbol{\sigma}_{\mu} \cdot \boldsymbol{p}
$$

is just the component of the polarization vector along the $\mu$-th axis. Unlike the strong measurements usually considered in quantum texts, where measurement of $\boldsymbol{\sigma}_{\mu}$ yields one of the random outcomes \pm 1 with probabilities $\left(1 \pm \boldsymbol{\sigma}_{\mu} \cdot \boldsymbol{p}\right) / 2$ and leaves the system in the corresponding state $\boldsymbol{p}= \pm \boldsymbol{\sigma}_{\mu}$, weak measurements of ensembleaverage expectation values can be made with only negligible perturbations to the ensemble as a whole [Per93]. This is in fact how quantum mechanical systems are usually manifest at the macroscopic level!

To see how all this relates to conventional wisdom, observe that the polarization vector of a pure state may be written in terms of the Cayley-Klein parameters as

$$
\boldsymbol{p}=2 \Re\left(\psi_{1}^{*} \psi_{2}\right) \boldsymbol{\sigma}_{\mathrm{x}}+2 \Im\left(\psi_{1}^{*} \psi_{2}\right) \boldsymbol{\sigma}_{\mathrm{y}}+\left(\left|\psi_{1}\right|^{2}-\left|\psi_{2}\right|^{2}\right) \boldsymbol{\sigma}_{\mathrm{z}} .
$$

Its stereographic projection from $-\sigma_{\mathrm{z}}$ onto the $\sigma_{\mathrm{x}} \sigma_{\mathrm{y}}$ plane is therefore

$$
\frac{2 \Re\left(\psi_{1}^{*} \psi_{2}\right) \sigma_{\mathrm{x}}+2 \Im\left(\psi_{1}^{*} \psi_{2}\right) \sigma_{\mathrm{y}}}{1+\left|\psi_{1}\right|^{2}-\left|\psi_{2}\right|^{2}} .
$$


Multiplying by $\sigma_{\times}$and simplifying the denominator using $\left|\psi_{1}\right|^{2}+\left|\psi_{2}\right|^{2}=1$ yields

$$
\frac{\Re\left(\psi_{1}^{*} \psi_{2}\right)+\Im\left(\psi_{1}^{*} \psi_{2}\right) \boldsymbol{K}}{\left|\psi_{1}\right|^{2}}
$$

where $\boldsymbol{K}=\sigma_{\mathrm{x}} \sigma_{\mathrm{y}}$ is a square-root of -1 . This is the same as the ratio $\psi_{2} / \psi_{1}$ save for the use of $\imath$ instead of $\boldsymbol{K}$ as the imaginary unit, which explains formally why $\mathrm{SO}(3)$ acts on the polarization vector in the same way that $\mathrm{SU}(2)$ acts on the ratio of the Cayley-Klein parameters [Alt86, FH81].

\section{Space-Time Geometry and Multiparticle Spinors}

The above interpretations apply only to single qubits (or to ensembles consisting of noninteracting and identical qubits). Extending them to systems of interacting and distinguishable qubits may be done in a physically significant fashion by considering the geometric algebra of space-time (or Minkowski space) $\mathfrak{R}_{1,3}$. This algebra, known as the Dirac algebra and denoted by $\mathcal{G}_{1,3}$, may be defined by the generating relations among an orthonormal basis analogous to Eq. (2.4):

$$
\begin{aligned}
& \gamma_{\mathrm{t}}^{2}=1, \quad \gamma_{\mu}^{2}=-1 \quad(\mu \in\{\mathrm{x}, \mathrm{y}, \mathrm{z}\}), \\
& \gamma_{\mu} \gamma_{\nu}=-\gamma_{\nu} \gamma_{\mu} \quad(\mu, \nu \in\{\mathrm{t}, \mathrm{x}, \mathrm{y}, \mathrm{z}\}, \mu \neq \nu)
\end{aligned}
$$

The corresponding geometric algebra separates into five inequivalent representations under the action of the full Lorentz group $\mathrm{O}(1,3)$, i.e.

$$
\begin{aligned}
\langle 1\rangle & \text { (scalars, 1-dimensional) } \\
\left\langle\gamma_{\mu}\right\rangle & \text { (vectors, 4-dimensional) } \\
\left\langle\gamma_{\mu} \gamma_{\nu}\right\rangle & \text { (bivectors, 6-dimensional) } \\
\left\langle\gamma_{\mu} \gamma_{\nu} \gamma_{\eta}\right\rangle & \text { (trivectors, 4-dimensional) } \\
\left\langle\gamma_{\mathrm{t}} \gamma_{\mathrm{x}} \gamma_{\mathrm{y}} \gamma_{\mathrm{z}}\right\rangle & \text { (pseudo-scalars, 1-dimensional) }
\end{aligned}
$$

where $\mu, \nu, \eta \in\{\mathrm{t}, \mathrm{x}, \mathrm{y}, \mathrm{z}\}$ with $\mu \neq \nu \neq \eta \neq \mu$, for a total dimension of 16 .

The important point for our purposes is that the even subalgebra of the Dirac algebra $\mathcal{G}_{1,3}^{+}$is isomorphic to the Pauli algebra $\mathcal{G}_{3}$ [Hes66]. This isomorphism may be constructed by choosing bases $\gamma_{\mu} \in \mathcal{G}_{1,3}$ and $\boldsymbol{\sigma}_{\mu} \in \mathcal{G}_{3}$, and defining an invertible linear mapping by

$$
\sigma_{\mu} \in \mathcal{G}_{3} \leftrightarrow \gamma_{\mu} \gamma_{\mathrm{t}} \in \mathcal{G}_{1,3}^{+} \quad(\mu \in\{\mathrm{x}, \mathrm{y}, \mathrm{z}\})
$$

These so-called relative spatial vectors $\gamma_{\mu} \gamma_{\mathrm{t}}$ satisfy the relations in Eq. (2.4), since

$$
\begin{aligned}
\left(\sigma_{\mu}\right)^{2} & \leftrightarrow\left(\gamma_{\mu} \gamma_{\mathrm{t}}\right)^{2}=-\gamma_{\mu}\left(\gamma_{\mathrm{t}}\right)^{2} \gamma_{\mu}=-\left(\gamma_{\mu}\right)^{2}=1 \\
\sigma_{\mu} \sigma_{\nu} & \leftrightarrow\left(\gamma_{\mu} \gamma_{\mathrm{t}}\right)\left(\gamma_{\nu} \gamma_{\mathrm{t}}\right)=\gamma_{\nu}\left(\gamma_{\mu} \gamma_{\mathrm{t}}\right) \gamma_{\mathrm{t}}=-\left(\gamma_{\nu} \gamma_{\mathrm{t}}\right)\left(\gamma_{\mu} \gamma_{\mathrm{t}}\right) \\
& \leftrightarrow-\sigma_{\nu} \sigma_{\mu} \quad(\mu, \nu \in\{\mathrm{x}, \mathrm{y}, \mathrm{z}\}, \mu \neq \nu)
\end{aligned}
$$

and hence generate an algebra isomorphic to $\mathcal{G}_{3}$. As bivectors in $\mathcal{G}_{1,3}$, however, they also generate $\mathcal{G}_{1,3}^{+}$, since

$$
\gamma_{\mu} \gamma_{\nu}=\gamma_{\mu}\left(\gamma_{\mathrm{t}}\right)^{2} \gamma_{\nu}=-\left(\gamma_{\mu} \gamma_{\mathrm{t}}\right)\left(\gamma_{\nu} \gamma_{\mathrm{t}}\right) \leftrightarrow-\sigma_{\mu} \sigma_{\nu}
$$

$(\mu, \nu \in\{\mathrm{x}, \mathrm{y}, \mathrm{z}\}, \mu \neq \nu)$, and similarly

$$
\iota \equiv \gamma_{\mathrm{t}} \gamma_{\mathrm{x}} \gamma_{\mathrm{y}} \gamma_{\mathrm{z}}=\left(\gamma_{\mathrm{x}} \gamma_{\mathrm{t}}\right)\left(\gamma_{\mathrm{y}} \gamma_{\mathrm{t}}\right)\left(\gamma_{\mathrm{z}} \gamma_{\mathrm{t}}\right) \leftrightarrow \sigma_{\mathrm{x}} \sigma_{\mathrm{y}} \sigma_{\mathrm{z}}
$$

Thus $\gamma_{\mu} \gamma_{\mathrm{t}} \leftrightarrow \sigma_{\mu}(\mu \in\{\mathrm{x}, \mathrm{y}, \mathrm{z}\})$ induces an algebra isomorphism as claimed, and when the bases are understood we may identify $\sigma_{\mu} \equiv \gamma_{\mu} \gamma_{\mathrm{t}}$. 
The choice of time-like vector $\gamma_{t} \in \mathfrak{R}_{1,3}$ in fact determines an inertial frame up to spatial rotation, in which the time $t$ and place $s$ of an event $\mathbf{e}$ in that frame are given by

$$
t+s=\mathbf{e} \cdot \gamma_{\mathrm{t}}+\mathbf{e} \wedge \gamma_{\mathrm{t}}=\mathbf{e} \gamma_{\mathrm{t}}
$$

(note that upright case is used for the space-time vector $\mathbf{e} \in \mathfrak{R}_{1,3}$ ). Thus the invariant interval between events separated by the space-time vector $\mathbf{e}$ is $\mathbf{e}^{2}=$ $\mathbf{e} \gamma_{\mathrm{t}}^{2} \mathbf{e}=(t+s)(t-s)=t^{2}-s^{2}$ as usual, while the relative velocity between events whose space-time velocities are $\gamma_{\mathrm{t}}$ and $\mathbf{v} \equiv \partial \mathbf{e} / \partial \tau$ is

$$
\boldsymbol{v}=\frac{\partial \boldsymbol{s}}{\partial t}=\frac{\partial \boldsymbol{s}}{\partial \tau} \frac{\partial \tau}{\partial t} \equiv\left(\frac{\partial \mathbf{e}}{\partial \tau} \wedge \gamma_{\mathrm{t}}\right)\left(\frac{\partial \mathbf{e}}{\partial \tau} \cdot \gamma_{\mathrm{t}}\right)^{-1}=\frac{\mathbf{v} \wedge \gamma_{\mathrm{t}}}{\mathbf{v} \cdot \gamma_{\mathrm{t}}}
$$

so that $\boldsymbol{v} \cdot \gamma_{\mathrm{t}}$ lies on an affine hyperplane in space-time.

A great deal of physics can be done in a manifestly Lorentz covariant fashion using the Dirac algebra. For example, the electromagnetic field at a given point in space-time corresponds to an arbitrary bivector $\mathbf{F} \in \bigwedge_{2} \Re_{1,3}$, called the Faraday bivector, and the covariant form of the Lorentz force equation is

$$
m \dot{\mathbf{v}}=q \mathbf{F} \cdot \mathbf{v}
$$

where $m$ is the rest mass, $q$ the charge and $\mathbf{v}$ the space-time velocity. (This is another example of the general rule that, in geometric algebra, the generators of motion are bivectors [DHSvA93].) The usual frame-dependent form is recovered by splitting the quantities in this equation by $\gamma_{\mathrm{t}}$ as above [Jan89]; in particular, the Faraday bivector splits into an electric and a magnetic field as $\mathbf{F} \equiv \boldsymbol{E}+\iota \boldsymbol{B}$, where

$$
\boldsymbol{E}=\left(\mathbf{F} \cdot \gamma_{\mathrm{t}}\right) \gamma_{\mathrm{t}} \text { and } \boldsymbol{\iota} \boldsymbol{B}=\left(\mathbf{F} \wedge \gamma_{\mathrm{t}}\right) \boldsymbol{\gamma}_{\mathrm{t}}
$$

The space-time reverse will be denoted by a tilde, e.g. in the present case $\tilde{\mathbf{F}}=-\mathbf{F}$. This is related to the spatial (or Pauli) reverse by $\mathbf{F}^{\dagger}=\boldsymbol{E}-\boldsymbol{\iota} \boldsymbol{B}=\boldsymbol{\gamma}_{\mathrm{t}} \tilde{\mathbf{F}} \boldsymbol{\gamma}_{\mathrm{t}}$. Both operations agree on the Pauli-even subalgebra, but the spatial reverse is not Lorentz coveriant since it depends on a particular $\gamma_{t}$.

Returning to our previous discussion of the density operator, we observe that the space-time form of the density operator of a single qubit polarized along $\mathrm{z}$ can be written as

$$
\boldsymbol{\rho}=\frac{1}{2}\left(1+\alpha \boldsymbol{\sigma}_{\mathrm{z}}\right)=\frac{1}{2}\left(\boldsymbol{\gamma}_{\mathrm{t}}+\alpha \gamma_{\mathrm{z}}\right) \boldsymbol{\gamma}_{\mathrm{t}} \equiv \boldsymbol{\varrho} \boldsymbol{\gamma}_{\mathrm{t}},
$$

where $-1 \leq \alpha \leq 1$ is the polarization and $\gamma_{\mathrm{t}}$ determines the local inertial frame. It follows that the Lorentz covariant form of the density operator is a time-like vector $\boldsymbol{\varrho} \in \mathfrak{R}_{1,3}$. Under a Lorentz boost $\boldsymbol{L}=\exp \left(-\lambda \boldsymbol{\sigma}_{\mathrm{z}} / 2\right) \in \mathrm{SO}(1,3)$ along $\boldsymbol{\sigma}_{\mathrm{z}}$, therefore, the relativistic density operator $\varrho$ transforms to $\boldsymbol{\varrho}^{\prime} \equiv \frac{1}{2} \boldsymbol{L}\left(\boldsymbol{\gamma}_{\mathrm{t}}+\alpha \gamma_{\mathrm{z}}\right) \tilde{\boldsymbol{L}}=$

$$
\frac{1}{2}\left(\cosh (\lambda) \gamma_{\mathrm{t}}-\sinh (\lambda) \gamma_{\mathrm{z}}+\alpha\left(\cosh (\lambda) \gamma_{\mathrm{z}}-\sinh (\lambda) \gamma_{\mathrm{t}}\right)\right) .
$$

This implies that in the unaccelerated frame (with renormalization by $\varrho^{\prime} \cdot \gamma_{\mathrm{t}}$ ),

$$
\begin{aligned}
\boldsymbol{\rho}^{\prime} & =\frac{\varrho^{\prime} \gamma_{\mathrm{t}}}{\boldsymbol{\varrho}^{\prime} \cdot \gamma_{\mathrm{t}}}=\frac{\underline{\varrho}^{\prime} \cdot \gamma_{\mathrm{t}}+\underline{\varrho}^{\prime} \wedge \gamma_{\mathrm{t}}}{\boldsymbol{\varrho}^{\prime} \cdot \gamma_{\mathrm{t}}} \\
& =\frac{1}{2}\left(1+\frac{\alpha \cosh (\lambda)-\sinh (\lambda)}{\cosh (\lambda)-\alpha \sinh (\lambda)} \sigma_{\mathrm{z}}\right) .
\end{aligned}
$$


It follows that the polarization itself transforms as

$$
\alpha^{\prime}=\frac{\alpha \cosh (\lambda)-\sinh (\lambda)}{\cosh (\lambda)-\alpha \sinh (\lambda)} .
$$

If we assume the qubit is at equilibrium with a heat bath, statistical mechanics tells us that $\alpha=\tanh (-\beta \epsilon / 2)$ where $\beta=1 /\left(k_{\mathrm{B}} T\right)$ is the inverse temperature and $\epsilon \in \mathbb{R}$ is the energy difference between the $|0\rangle$ and $|1\rangle$ states [Tol38]. Then the addition formulae for cosh and sinh give

$$
\alpha^{\prime}=\tanh (-\beta \epsilon / 2-\lambda),
$$

so the apparent equilibrium polarization depends on velocity. These results are not to be found in the classic treatise on relativistic thermodynamics [Tol34].

We will now construct a Lorentz covariant multiparticle theory of qubit systems in the simplest possible way, by taking a direct sum of copies of space-time (regarded as a vector space, rather than an algebra), one for each of the $N$ qubits, i.e.

$$
\bigoplus_{q=1}^{N}\left\langle\gamma_{\mathrm{t}}^{q}, \gamma_{\mathrm{x}}^{q}, \gamma_{\mathrm{y}}^{q}, \gamma_{\mathrm{z}}^{q}\right\rangle
$$

and considering the associated geometric algebra $\mathcal{G}_{N, 3 N}$. Then the even subalgebras of different particle spaces $p \neq q$ commute, since (in any given bases)

$$
\boldsymbol{\sigma}_{\mu}^{p} \boldsymbol{\sigma}_{\nu}^{q}=\gamma_{\mu}^{p}\left(\gamma_{\nu}^{q} \gamma_{\mathrm{t}}^{q}\right) \gamma_{\mathrm{t}}^{p}=\boldsymbol{\sigma}_{\nu}^{q} \boldsymbol{\sigma}_{\mu}^{p}
$$

for all $\mu, \nu \in\{\mathrm{x}, \mathrm{y}, \mathrm{z}\}$, so that the algebra generated by the even subalgebras is isomorphic to a tensor product of these algebras, written as

$$
\left(\mathcal{G}_{1,3}^{+}\right)^{\otimes N} \approx \mathcal{G}_{3}^{\otimes N} \equiv\left(\mathcal{G}_{3}\right)^{\otimes N} \text {. }
$$

This construction of the tensor product was first used by Clifford as a means of studying the tensor products of quaternion algebras [Cli78]; van der Waerden has in fact called it a Clifford algebra of the second kind [vdW85]. As a means of justifying the tensor product of nonrelativistic quantum mechanics in terms of the underlying geometry of space-time, however, it is a much more recent development [DLG93].

A key feature of quantum mechanics, which is needed for quantum computers to be able to solve problems more efficiently than their classical counterparts, is an exponential growth in the dimension of the Hilbert space of a multi-qubit system with the number of particles involved. The complex dimension of the Hilbert space $(\mathfrak{H})^{\otimes N}$ of an $N$-qubit system is in fact $2^{N}$, and the space of operators (linear transformations) on $(\mathfrak{H})^{\otimes N}$ therefore has real dimension $2^{2 N+1}$. The above construction yields a space of "operators" $\mathcal{G}_{3}^{\otimes N}$ whose real dimension also grows exponentially, but as $2^{3 N}$. The extra degrees of freedom are due to the presence of a different unit pseudo-scalar $\iota^{q}$ in every particle space. They can easily be removed by multiplying through by an idempotent element called the correlator:

$$
\boldsymbol{C} \equiv \frac{1}{2}\left(1-\iota^{1} \iota^{2}\right) \frac{1}{2}\left(1-\iota^{1} \iota^{3}\right) \cdots \frac{1}{2}\left(1-\iota^{1} \iota^{N}\right)
$$

This commutes with everything in $\mathcal{G}_{3}^{\otimes N}$ and satisfies $\iota^{p} \iota^{q} \boldsymbol{C}=-\boldsymbol{C}$ for $1 \leq p, q \leq N$, so that multiplication by it homomorphically maps $\mathcal{G}_{3}^{\otimes N}$ onto an ideal $\mathcal{G}_{3}^{\otimes N} / \boldsymbol{C}$ wherein all the unit pseudo-scalars have been identified, ${ }^{2}$ and which therefore has

\footnotetext{
${ }^{2}$ The notation $\mathcal{G}_{3}^{\otimes N} / \boldsymbol{C}$ is justified by the fact that the two-sided principle ideal $\mathcal{G}_{3}^{\otimes N}(\boldsymbol{C})$ generated by $\boldsymbol{C}$ is isomorphic to the quotient algebra $\mathcal{G}_{3}^{\otimes N} / \operatorname{ker}(\boldsymbol{C})$, where $\operatorname{ker}(\boldsymbol{C}) \equiv\left\{\boldsymbol{g} \in \mathcal{G}_{3}^{\otimes N} \mid\right.$ $\boldsymbol{g} \boldsymbol{C}=0\}$.
} 
the correct dimension over $\mathbb{R}$. As a subalgebra, this ideal is in fact isomorphic to the algebra of $2^{N} \times 2^{N}$ complex matrices, and hence capable of describing all the states and transformations of (ensembles of) $N$ qubit systems. In the following, we shall generally omit $\boldsymbol{C}$ from our expressions altogether, and use a single unit imaginary $\iota$ as in conventional quantum mechanics.

On the "even" subalgebra $\left(\mathcal{G}_{3}^{+}\right)^{\otimes N}$, multiplication by the correlator turns out to be an algebra automorphism; this algebra can thus be written as

$$
\left(\mathcal{G}_{3}^{+}\right)^{\otimes N} \approx\left(\mathcal{G}_{3}^{+}\right)^{\otimes N} / \boldsymbol{C} \approx\left(\mathcal{G}_{3}^{\otimes N} / \boldsymbol{C}\right)^{+} \approx \mathcal{S U}(2)^{\otimes N}
$$

where the "+" refers throughout to the subalgebra generated by expressions which are invariant under inversion in the origin, and $\mathcal{S U}(2)^{\otimes N}$ to the algebra generated over $\mathbb{R}$ by the Kronecker products of matrices in the group $\mathrm{SU}(2)$. This subalgebra has real dimension $2^{2 N}$, but is mapped onto a left-ideal of dimension $2^{N+1}$ by right-multiplication with another idempotent which is given by the tensor product of those considered earlier, namely

$$
\boldsymbol{E}_{+} \equiv \boldsymbol{E}_{+}^{1} \boldsymbol{E}_{+}^{2} \cdots \boldsymbol{E}_{+}^{N}
$$

where $\boldsymbol{E}_{ \pm}^{q} \equiv\left(1 \pm \boldsymbol{\sigma}_{\mathrm{z}}^{q}\right) / 2$ for $q=1, \ldots, N$. Henceforth, the term "even subalgebra" will refer to $\left(\mathcal{G}_{3}^{+}\right)^{\otimes N}$ (suitably correlated) unless otherwise stated.

In terms of the usual matrix representation, right-multiplication of an element of the even subalgebra $\underline{\boldsymbol{\Psi}}$ by $\underline{\boldsymbol{E}}$ + likewise sets all but the first column to zero, so that $\boldsymbol{\Psi} \boldsymbol{E}_{+}$transforms like a "spinor" in $\mathfrak{H}^{\otimes N}$ under left-multiplication by single particle rotations $\boldsymbol{R}^{q} \in\left(\mathcal{G}_{3}^{+}\right)^{\otimes N}$. Unlike the single particle case, however, this one column does not uniquely determine an element of the even subalgebra $\left(\mathcal{G}_{3}^{+}\right)^{\otimes N} / \boldsymbol{C}$. What has been proposed instead [DLG93] is to use the fact that $\boldsymbol{E}_{+}$"absorbs" $\boldsymbol{\sigma}_{\mathbf{z}}$ 's to distribute copies of the latter across the correlator, converting it to what will here be called the directional correlator $\boldsymbol{D}$, i.e.

$$
\boldsymbol{\Psi} C \boldsymbol{E}_{+}=\boldsymbol{\Psi} \boldsymbol{C}\left(\left(\sigma_{\mathrm{z}}^{1}\right)^{N-1} \boldsymbol{\sigma}_{\mathrm{z}}^{2} \cdots \boldsymbol{\sigma}_{\mathrm{z}}^{N}\right) \boldsymbol{E}_{+}=\boldsymbol{\Psi} \boldsymbol{D} \boldsymbol{E}_{+},
$$

where

$$
\boldsymbol{D} \equiv \frac{1}{2}\left(1-\iota^{1} \boldsymbol{\sigma}_{\mathrm{z}}^{1} \iota^{2} \boldsymbol{\sigma}_{\mathrm{z}}^{2}\right) \frac{1}{2}\left(1-\boldsymbol{\iota}^{1} \boldsymbol{\sigma}_{\mathrm{z}}^{1} \iota^{3} \boldsymbol{\sigma}_{\mathrm{z}}^{3}\right) \cdots \frac{1}{2}\left(1-\boldsymbol{\iota}^{1} \boldsymbol{\sigma}_{\mathrm{z}}^{1} \iota^{N} \boldsymbol{\sigma}_{\mathrm{z}}^{N}\right) .
$$

It can be shown that right-multiplication by $\boldsymbol{D}$, unlike $\boldsymbol{C}$, reduces the dimensionality to $2^{N+1}$, thereby permitting the objects in this reduced even subalgebra $\left(\mathcal{G}_{3}^{+}\right)^{\otimes N} / \boldsymbol{D}$ to be regarded as spinors, analogous to $\mathcal{G}_{3}^{+}$for a single qubit. In the corresponding left-ideal, $\boldsymbol{K} \equiv \boldsymbol{\iota}^{1} \boldsymbol{\sigma}_{\mathrm{z}}^{1} \boldsymbol{D} \leftrightarrow \cdots \leftrightarrow \boldsymbol{\iota}^{N} \boldsymbol{\sigma}_{\mathrm{z}}^{N} \boldsymbol{D}$ serves as the unit imaginary, since $\boldsymbol{K}^{2}=-\boldsymbol{D}$, but is required to always operate from the right. Henceforth, unless otherwise mentioned, we will regard spinors $\left(\mathcal{G}_{3}^{+}\right)^{\otimes N} / \boldsymbol{D}=\left(\left(\mathcal{G}_{3}^{+}\right)^{\otimes N} / \boldsymbol{C}\right) /(\boldsymbol{C D})$ as a left-ideal in the $\boldsymbol{C}$-correlated even subalgebra, drop both $\boldsymbol{C}$ and the superscripts on the $\boldsymbol{\iota}$ 's as above, and use $\boldsymbol{D}$ as a short-hand for $\boldsymbol{C D}=\boldsymbol{D} \boldsymbol{C}$.

In the case of two qubits, for example, the identifications are induced by $\boldsymbol{D}$ are

$$
\begin{array}{rrrr}
|00\rangle & -1 \stackrel{\boldsymbol{D}}{\longleftrightarrow} \iota \sigma_{\mathrm{z}}^{1} \iota \sigma_{\mathrm{z}}^{2} & \boldsymbol{\iota} \sigma_{\mathrm{z}}^{1} \stackrel{\boldsymbol{D}}{\longleftrightarrow} \iota \sigma_{\mathrm{z}}^{2} \\
|01\rangle & \boldsymbol{\iota} \sigma_{\mathrm{y}}^{2} \stackrel{\boldsymbol{D}}{\longleftrightarrow} \iota \sigma_{\mathrm{z}}^{1} \iota \sigma_{\mathrm{x}}^{2} & -\iota \sigma_{\mathrm{x}}^{2} \stackrel{\boldsymbol{D}}{\longleftrightarrow} \iota \sigma_{\mathrm{z}}^{1} \iota \sigma_{\mathrm{y}}^{2} \\
|10\rangle & \iota \sigma_{\mathrm{y}}^{1} \stackrel{\boldsymbol{D}}{\longleftrightarrow} \iota \sigma_{\mathrm{x}}^{1} \iota \sigma_{\mathrm{z}}^{2} & -\iota \sigma_{\mathrm{x}}^{1} \stackrel{\boldsymbol{D}}{\longleftrightarrow} \iota \sigma_{\mathrm{y}}^{1} \iota \sigma_{\mathrm{z}}^{2} \\
|11\rangle & -\boldsymbol{\iota} \sigma_{\mathrm{y}}^{1} \iota \sigma_{\mathrm{y}}^{2} \stackrel{\boldsymbol{D}}{\longleftrightarrow} \iota \sigma_{\mathrm{x}}^{1} \iota \sigma_{\mathrm{x}}^{2} & \iota \sigma_{\mathrm{x}}^{1} \iota \sigma_{\mathrm{y}}^{2} \stackrel{\boldsymbol{D}}{\longleftrightarrow} \iota \sigma_{\mathrm{y}}^{1} \iota \sigma_{\mathrm{x}}^{2}
\end{array}
$$


(where the two columns differ by operation with $\boldsymbol{K}$ ). ¿From this it may be seen that any "spinor" in $\left(\mathcal{G}_{3}^{+}\right)^{\otimes 2} / \boldsymbol{D}$ can be written as

$$
\begin{aligned}
\boldsymbol{\Psi}= & \left(\left(\alpha_{0}+\beta_{0} \boldsymbol{K}\right)-\iota \sigma_{\mathrm{y}}^{2}\left(\alpha_{1}+\beta_{1} \boldsymbol{K}\right)-\right. \\
& \left.\boldsymbol{\iota} \sigma_{\mathrm{y}}^{1}\left(\alpha_{2}+\beta_{2} \boldsymbol{K}\right)+\iota \sigma_{\mathrm{y}}^{1} \iota \sigma_{\mathrm{y}}^{2}\left(\alpha_{3}+\beta_{3} \boldsymbol{K}\right)\right) \boldsymbol{D}
\end{aligned}
$$

(cf. [SLD99]). Alternatively, again using Eq. (3.24), a unit norm spinor may be factorized into a product of entities in the correlated and reduced even subalgebra, namely $\boldsymbol{\Psi}=\boldsymbol{R}^{1} \boldsymbol{S}^{2} \boldsymbol{T} \boldsymbol{P} \boldsymbol{D} \boldsymbol{C}$, where

$$
\begin{array}{rlrl}
\boldsymbol{R}^{1} & \equiv e^{-\boldsymbol{\iota} \phi \boldsymbol{\sigma}_{\mathrm{z}}^{1} / 2} e^{-\boldsymbol{\iota} \theta \boldsymbol{\sigma}_{\mathrm{y}}^{1} / 2}, & & \boldsymbol{T} \equiv \cos (\varsigma / 2)-\sin (\varsigma / 2) \boldsymbol{\sigma}_{\mathrm{y}}^{1} \boldsymbol{\sigma}_{\mathrm{y}}^{2} \boldsymbol{K}, \\
\boldsymbol{S}^{2} \equiv e^{-\boldsymbol{\iota} \varphi \boldsymbol{\sigma}_{\mathrm{z}}^{2} / 2} e^{-\boldsymbol{\iota} \vartheta \boldsymbol{\sigma}_{\mathrm{y}}^{2} / 2}, & \boldsymbol{P} \equiv e^{-\tau \boldsymbol{K} / 2} .
\end{array}
$$

Thus when $\varsigma=\pi$, the factor $\boldsymbol{T}$ becomes

$$
-\boldsymbol{\sigma}_{\mathrm{y}}^{1} \boldsymbol{\sigma}_{\mathrm{y}}^{2} \boldsymbol{K}=\left(-\iota \boldsymbol{\sigma}_{\mathrm{y}}^{1}\right)\left(-\iota \boldsymbol{\sigma}_{\mathrm{y}}^{2}\right) \boldsymbol{K}=e^{-(\pi / 2) \iota \sigma_{\mathrm{y}}^{1}} e^{-(\pi / 2) \iota \sigma_{\mathrm{y}}^{2}} \boldsymbol{K},
$$

so that the arguments of the exponentials involving $\boldsymbol{\iota} \boldsymbol{\sigma}_{\mathrm{y}}^{1}$ and $\boldsymbol{\iota} \boldsymbol{\sigma}_{\mathrm{y}}^{2}$ in the first two factors are shifted by $\pi / 2$ while the total phase is shifted by $\tau=\pi$. It follows that $\boldsymbol{T}$ rotates the first two factors in the planes defined by their conjugate spinors $\left[-r_{2}^{*} ; r_{1}^{*}\right],\left[-s_{2}^{*} ; s_{1}^{*}\right]$. Thus on right-multiplying by $\boldsymbol{E}_{+}$and expanding in the usual basis, we obtain (up to an overall phase)

$$
\begin{aligned}
\underline{\boldsymbol{\Psi}}= & \cos \left(\frac{\varsigma}{2}\right) e^{\imath \tau / 2}\left[\begin{array}{c}
\cos \left(\frac{\theta}{2}\right) e^{\imath \phi / 2} \\
\sin \left(\frac{\theta}{2}\right) e^{-\imath \phi / 2}
\end{array}\right] \otimes\left[\begin{array}{c}
\cos \left(\frac{\vartheta}{2}\right) e^{\imath \varphi / 2} \\
\sin \left(\frac{\vartheta}{2}\right) e^{-\imath \varphi / 2}
\end{array}\right]+ \\
& \sin \left(\frac{\varsigma}{2}\right) e^{-\imath \tau / 2}\left[\begin{array}{c}
\sin \left(\frac{\theta}{2}\right) e^{\imath \phi / 2} \\
-\cos \left(\frac{\theta}{2}\right) e^{-\imath \phi / 2}
\end{array}\right] \otimes\left[\begin{array}{c}
\sin \left(\frac{\vartheta}{2}\right) e^{\imath \varphi / 2} \\
-\cos \left(\frac{\vartheta}{2}\right) e^{-\imath \varphi / 2}
\end{array}\right]
\end{aligned}
$$

This is known as the Schmidt decomposition [EK95]. It is useful in studying the entanglement of bipartite quantum systems, which (in conventional terms) means that $|\psi\rangle \in \mathfrak{H}^{\otimes 2}$ cannot be written as a product $\left|\psi^{1}\right\rangle \otimes\left|\psi^{2}\right\rangle \equiv\left|\psi^{1}\right\rangle\left|\psi^{2}\right\rangle \equiv\left|\psi^{1} \psi^{2}\right\rangle$ for any one-particle spinors $\left|\psi^{1}\right\rangle,\left|\psi^{2}\right\rangle \in \mathfrak{H}$. In fact it is just the singular value decomposition in disguise, since (for example) on arranging the entries of a twoqubit spinor $|\psi\rangle=\left[\psi_{1} ; \cdots ; \psi_{4}\right]$ in a $2 \times 2$ matrix, we can write

$$
\underline{\boldsymbol{\Psi}} \equiv\left[\begin{array}{ll}
\psi_{1} & \psi_{3} \\
\psi_{2} & \psi_{4}
\end{array}\right]=\underline{\boldsymbol{U}} \underline{\boldsymbol{V}} \underline{\boldsymbol{W}}^{\dagger}=\underline{\boldsymbol{u}}^{1} v^{11}\left(\underline{\boldsymbol{w}}^{1}\right)^{\dagger}+\underline{\boldsymbol{u}}^{2} v^{22}\left(\underline{\boldsymbol{w}}^{2}\right)^{\dagger},
$$

where $\underline{\boldsymbol{V}}$ is a $2 \times 2$ diagonal matrix containing the singular values $v^{11} \geq v^{22} \geq 0$ and $\underline{\boldsymbol{U}}, \underline{\boldsymbol{W}}$ are unitary matrices with columns $\underline{\boldsymbol{u}}^{k}, \underline{\boldsymbol{w}}^{k}$, respectively. Since the entries of the dyadic products $\underline{\boldsymbol{u}}^{1}\left(\underline{\boldsymbol{w}}^{1}\right)^{\dagger}, \underline{\boldsymbol{u}}^{2}\left(\underline{\boldsymbol{w}}^{2}\right)^{\dagger}$ are exactly the same as the Kronecker matrix products $\underline{\boldsymbol{u}}^{1} \otimes \underline{\boldsymbol{w}}^{1}, \underline{\boldsymbol{u}}^{2} \otimes \underline{\boldsymbol{w}}^{2}$, the equivalence with Eq. (3.28) follows with $v^{11} \equiv \cos (\varsigma / 2), v^{22} \equiv \sin (\varsigma / 2)$, and the Kronecker products of the columns of $\underline{\boldsymbol{U}}$ and $\underline{\boldsymbol{W}}$ identified with conjugate pairs of single qubit spinors whose relative phases are given by $\exp ( \pm \imath \tau / 2)$.

Clearly a two-qubit spinor is unentangled if and only if $v^{11}=1$, which is equivalent to $\varsigma=0$ or $\boldsymbol{T}=1$. Thus $\boldsymbol{T}$ describes the entanglement of the qubits, and is accordingly called the tangler. The geometric algebra approach clearly provides deeper insight into the structure of entanglement than does one based on mechanical matrix algebra. In particular, the fact that $\tilde{\boldsymbol{\Psi}} \boldsymbol{\Psi}$ is even and reversion-symmetric 
in the Dirac as well as the Pauli algebra implies that it is the sum of a scalar and a four-vector in the two-particle Dirac algebra $\mathcal{G}_{2,6}$. Since Lorentz transformations of the spinors cancel, this entity is in fact a Lorentz invariant, and dividing out the total phase $\boldsymbol{P}$ as $\boldsymbol{P}(\tilde{\boldsymbol{\Psi}} \boldsymbol{\Psi}) \tilde{\boldsymbol{P}}$ yields the square of the tangler directly. The availability of such powerful methods of manipulating entities in the multiparticle Dirac algebra promises to be useful in finding analogs of the Schmidt decomposition for three or more qubits.

\section{Quantum Operations on Density Operators}

Quantum computers operate on information stored in the states of quantum systems. The systems are usually assumed to be arrays of distinguishable qubits (two-state subsystems), whose basis states $|0\rangle$ and $|1\rangle$ correspond to the binary digits 0 and 1 , respectively, while the operations are usually taken to be unitary. General unitary transformations of the qubits are built up from simpler ones that affect only a few qubits at a time, which are called quantum logic gates. The representation of these gates in suitable products of Clifford algebras has been described in Refs. [SCH98, Vla01]. The goal here will be to show how gates act upon spinors in the even subalgebra, and how they can be extended to a wider class of nonunitary quantum operations on density operators.

Given the isomorphism between the algebra of $2^{N} \times 2^{N}$ matrices over $\mathbb{C}$ and $\mathcal{G}_{3}^{\otimes N} / \boldsymbol{C}$ relative to a choice of basis in each particle space, it is straightforward to interpret matrices in the former as geometric entities in the latter. A matrix $\boldsymbol{U} \in \mathrm{U}\left(2^{N}\right)$, however, does not generally correspond to an entity $\boldsymbol{U}$ in the even subalgebra $\left(\mathcal{G}_{3}^{+}\right)^{\otimes N} / \boldsymbol{C}$, so that $\boldsymbol{U} \boldsymbol{\Psi} \notin\left(\mathcal{G}_{3}^{+}\right)^{\otimes N} / \boldsymbol{D}$ for a general spinor $\boldsymbol{\Psi} \in\left(\mathcal{G}_{3}^{+}\right)^{\otimes N} / \boldsymbol{D}$. Nevertheless, letting $\boldsymbol{E}_{-} \equiv \prod_{q} \boldsymbol{E}_{-}^{q}$ be the idempotent "opposite" to $\boldsymbol{E}_{+}$, and noting that this satisfies $\boldsymbol{E}_{+} \boldsymbol{E}_{-}=0$, the product of $\boldsymbol{U} \boldsymbol{\Psi}$ with $\boldsymbol{E}_{+}$may be written as

$$
\boldsymbol{U} \boldsymbol{\Psi} \boldsymbol{E}_{+}=\left(\boldsymbol{U} \boldsymbol{\Psi} \boldsymbol{E}_{+}+\hat{\boldsymbol{U}} \boldsymbol{\Psi} \boldsymbol{E}_{-}\right) \boldsymbol{E}_{+}=2\left\langle\boldsymbol{U} \boldsymbol{\Psi} \boldsymbol{E}_{+}\right\rangle_{+} \boldsymbol{E}_{+},
$$

where the "hat" on $\hat{\boldsymbol{U}}$ denotes its image under inversion in the origin (so that $\hat{\boldsymbol{E}}_{+}=\boldsymbol{E}_{-}$), and hence $\left\langle_{-}\right\rangle_{+}$is a projection onto the even subalgebra. Because $\left(\mathcal{G}_{3}^{+}\right)^{\otimes N} / \boldsymbol{C}$ and $\mathrm{U}\left(2^{N}\right)$ are both $\left(2^{2 N}\right)$-dimensional, nothing is lost in this projection! Thus we can drop the right-factor of $\boldsymbol{E}_{+}$as usual, and define the action of $\boldsymbol{U}$ on $\boldsymbol{\Psi} \in\left(\mathcal{G}_{3}^{+}\right)^{\otimes N} / \boldsymbol{D}$ as

$$
\boldsymbol{U} \circ \boldsymbol{\Psi} \equiv 2\left\langle\boldsymbol{U} \boldsymbol{\Psi} \boldsymbol{E}_{+}\right\rangle_{+} \cdot
$$

More generally, the usual action of the Pauli matrices on spinors corresponds to the following action of the basis vectors on the reduced even subalgebra [DLG93]:

$$
\sigma_{\mu} \circ \Psi \equiv \sigma_{\mu} \Psi \sigma_{\mathrm{z}}, \quad \iota \circ \Psi \equiv \iota \Psi \sigma_{\mathrm{z}}
$$

The simplest logic gate is the NOT of a single qubit, which operates on the computational basis as follows:

$$
\underline{\boldsymbol{N}}|0\rangle=|1\rangle \leftrightarrow-\iota \sigma_{\mathrm{y}}, \quad \underline{\boldsymbol{N}}|1\rangle=|0\rangle \leftrightarrow 1
$$

Thus it might appear reasonable to represent the NOT by $\boldsymbol{N} \equiv \iota \sigma_{\mathrm{y}} \in \mathrm{SU}(2)$, but when $\iota \sigma_{\mathrm{y}}$ is applied a superposition $\left(1-\iota \sigma_{\mathrm{y}}\right) / \sqrt{2} \leftrightarrow(|0\rangle+|1\rangle) / \sqrt{2}$, we get $(1+$ $\left.\iota \sigma_{\mathrm{y}}\right) / \sqrt{2} \leftrightarrow(|0\rangle-|1\rangle) / \sqrt{2}$ instead of $(|0\rangle+|1\rangle) / \sqrt{2}$ again. For a single qubit this difference is just an overall rotation by $\pi$ about $\boldsymbol{\sigma}_{\mathbf{z}}$, but a second qubit can be affected by this phase difference between the first qubit's states. Therefore the correct 
representation of the NOT gate in $\mathrm{SU}(2)$ is actually $\boldsymbol{N} \equiv \pm \boldsymbol{\iota} \sigma_{\mathrm{x}}$, which preserves this superposition up to an irrelevant overall phase shift: $\underline{\boldsymbol{\sigma}}_{\times}(|0\rangle+|1\rangle) / \sqrt{2} \leftrightarrow$

$$
\begin{aligned}
& \left(\iota \sigma_{\mathrm{x}}\right) \circ\left(1-\iota \sigma_{\mathrm{y}}\right) / \sqrt{2}=\iota \circ \sigma_{\mathrm{x}} \circ\left(1-\iota \sigma_{\mathrm{y}}\right) / \sqrt{2} \\
= & \iota \circ\left(\sigma_{\mathrm{x}}\left(1-\iota \sigma_{\mathrm{y}}\right) \sigma_{\mathrm{z}} / \sqrt{2}\right)=\iota \circ\left(-\iota \sigma_{\mathrm{y}}+1\right) / \sqrt{2} \\
= & \left(-\iota \sigma_{\mathrm{y}}+1\right) \iota \sigma_{\mathrm{z}} / \sqrt{2} \leftrightarrow-\imath(|0\rangle+|1\rangle) / \sqrt{2}
\end{aligned}
$$

More interesting logical operations on the qubits must be able to transform the state of one conditional on that of another. The usual way in which this is done is via the c-NOT or controlled-NOT gate. As a matrix in SU(4), this is represented in the computational basis by

$$
\underline{\boldsymbol{N}}^{2 \mid 1} \equiv \sqrt{\imath}\left[\begin{array}{cccc}
1 & 0 & 0 & 0 \\
0 & 1 & 0 & 0 \\
0 & 0 & 0 & 1 \\
0 & 0 & 1 & 0
\end{array}\right],
$$

which makes it clear that this operation NOT's the second qubit whenever the first is 1 . The corresponding operator in geometric algebra is

$$
\boldsymbol{N}^{2 \mid 1} \equiv\left(1+\iota \sigma_{\mathrm{z}}^{1}\right) / \sqrt{2}\left(\boldsymbol{E}_{+}^{1}+\boldsymbol{E}_{-}^{1} \iota \sigma_{\times}^{2}\right) .
$$

This may also be written in exponential form as

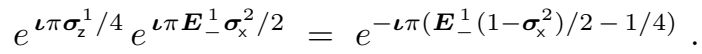

Physical implementations of this operation by e.g. NMR typically expand the exponential into a product of relatively simple commuting factors which can be performed sequentially [HSTC00].

Note that since $\left(1-\sigma_{\times}^{2}\right) / 2$ is also an idempotent, $\boldsymbol{N}^{2 \mid 1}$ differs from $\boldsymbol{N}^{1 / 2}$ by a swap of the $x$ and $z$ axes for both qubits. This self-inverse operation, called the Hadamard transform $\boldsymbol{H}$, is simply a rotation by $\pi$ about the $\left(\boldsymbol{\sigma}_{\mathrm{x}}+\boldsymbol{\sigma}_{\mathrm{z}}\right) / \sqrt{2}$ axis. Sandwiching $\boldsymbol{N}^{2 \mid 1}$ by Hadamards $\boldsymbol{H}^{2}=\boldsymbol{\iota}\left(\boldsymbol{\sigma}_{\mathrm{x}}^{2}+\boldsymbol{\sigma}_{\mathrm{z}}^{2}\right) / \sqrt{2}$ to just the second qubit gives

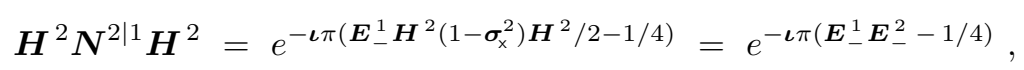

so the c-NOT can also be viewed as a rotated phase shift of the state $|11\rangle$ by $\pi$. The Hadamard gate has the important feature of transforming basis states into superpositions thereof; indeed, as an element of the even subalgebra, it actually represents the spinor of a uniform superposition directly:

$$
\begin{aligned}
& \underline{\boldsymbol{H}}|0\rangle=\imath(|0\rangle+|1\rangle) / \sqrt{2} \leftrightarrow\left(\boldsymbol{\iota} \sigma_{\mathrm{z}}+\boldsymbol{\iota} \sigma_{\mathrm{x}}\right) / \sqrt{2} \boldsymbol{D} \\
& \underline{\boldsymbol{H}}|1\rangle=\imath(|0\rangle-|1\rangle) / \sqrt{2} \leftrightarrow\left(\boldsymbol{\iota} \sigma_{\mathrm{z}}-\boldsymbol{\iota} \sigma_{\mathrm{x}}\right) / \sqrt{2} \boldsymbol{D}
\end{aligned}
$$

Thus, by using the relations (4.3), we can show that applying a Hadamard to one of two qubits in the state $|11\rangle$ followed by a c-NOT gate to the other yields the entangled singlet state: $\underline{\boldsymbol{N}}^{2 \mid 1} \underline{\boldsymbol{H}}^{1}|11\rangle \leftrightarrow$

$$
\begin{aligned}
& \frac{1}{2}\left((1+\iota) \boldsymbol{E}_{+}^{1}+(1-\iota) \boldsymbol{E}_{-}^{1} \iota \sigma_{\mathrm{x}}^{2}\right) \circ\left(\left(\iota \sigma_{\mathrm{z}}^{1}-\boldsymbol{\iota} \sigma_{\mathrm{x}}^{1}\right)\left(-\iota \sigma_{\mathrm{y}}^{2}\right)\right) \\
= & \frac{1}{2}\left(\left((1+\iota) \circ\left(\iota \sigma_{\mathrm{z}}^{1}\right)\right)\left(-\iota \sigma_{\mathrm{y}}^{2}\right)+\left((1-\iota) \circ\left(-\iota \sigma_{\mathrm{x}}^{1}\right)\right)\left(\left(\iota \sigma_{\mathrm{x}}^{2}\right) \circ\left(-\iota \sigma_{\mathrm{y}}^{2}\right)\right)\right) \\
= & \frac{1}{2}\left(1-\iota \sigma_{\mathrm{z}}^{1}\right) \iota \sigma_{\mathrm{y}}^{2}-\frac{1}{2}\left(\iota \sigma_{\mathrm{x}}^{1}-\iota \sigma_{\mathrm{y}}^{1}\right) \iota \sigma_{\mathrm{z}}^{2} \stackrel{D}{\longrightarrow} \frac{\iota}{2}\left(\sigma_{\mathrm{y}}^{2}+\sigma_{\mathrm{x}}^{2}-\sigma_{\mathrm{y}}^{1}-\sigma_{\mathrm{x}}^{1}\right)
\end{aligned}
$$


$\leftrightarrow \sqrt{-\imath}(|10\rangle-|01\rangle) / \sqrt{2} \equiv \sqrt{-\imath}\left|\psi_{-}\right\rangle$. "Quantum" gates like $\boldsymbol{H}$ are not, of course, found in conventional boolean logic, and are an essential component of all quantum algorithms that are more efficient than their classical counterparts [EJ98, CEMM98]. Indeed, the c-NOT gate together with general single qubit rotations are known to generate $\mathrm{SU}\left(2^{N}\right)$, and hence are universal for quantum $\operatorname{logic}\left[\mathbf{B B C}^{+} \mathbf{9 5}\right]$.

It turns out that unitary transformations are not the most general sort of operation that can be applied to a quantum system. Most such quantum operations, however, produce a statistical outcome, and the ensemble of possible outcomes must be described by a density operator. The previous definition (Eq. (2.20)) of the density operator of an ensemble of identical and noninteracting qubits may be extended to an ensemble of multi-qubit systems as follows:

$$
\underline{\boldsymbol{\rho}} \equiv \overline{|\psi\rangle\langle\psi|} \leftrightarrow \boldsymbol{\rho} \equiv \overline{(\boldsymbol{\Psi} \boldsymbol{D}) \boldsymbol{E}_{+}(\boldsymbol{\Psi} \boldsymbol{D})^{\sim}}=\overline{\boldsymbol{\Psi} \boldsymbol{E}_{+} \tilde{\boldsymbol{\Psi}} \boldsymbol{C}}
$$

Suppressing the correlator $\boldsymbol{C}$ as usual, $\boldsymbol{\rho}$ may also be expressed in diagonal form as

$$
\boldsymbol{\rho}=\boldsymbol{R}\left(\sum_{k=0}^{2^{N}} \rho_{k} \boldsymbol{E}_{(k)}\right) \boldsymbol{R}^{\dagger}=\sum_{k=0}^{2^{N}} \rho_{k} \boldsymbol{r}_{k} \boldsymbol{r}_{k}^{\dagger} .
$$

where $\boldsymbol{R} \in \mathcal{G}_{3}^{\otimes N} / \boldsymbol{C}$ corresponds to a unitary matrix $\underline{\boldsymbol{R}} \in \mathrm{U}\left(2^{N}\right)$ (in the usual $\boldsymbol{\sigma}_{\mathrm{z}}$ coordinate system), and $0 \leq \rho_{k} \leq 1$ are the eigenvalues of $\boldsymbol{\rho}$. The idempotents $\boldsymbol{E}_{(k)}$ are given by $\prod_{q} \boldsymbol{E}_{\epsilon_{k}^{q}}^{q} \leftrightarrow\left|\chi_{k}^{1} \cdots \chi_{k}^{N}\right\rangle\left\langle\chi_{k}^{1} \cdots \chi_{k}^{N}\right|$, where $\epsilon_{k}^{q} \equiv 1-2 \chi_{k}^{q}$ with $\chi_{k}^{q}$ equal to the $q$-th bit in the binary expansion of $k \in\left\{0, \ldots, 2^{N}-1\right\}$. It follows that $\left|\rho_{k}\right\rangle \leftrightarrow \boldsymbol{r}_{k} \equiv \boldsymbol{R} \boldsymbol{E}_{(k)}$ for $\rho_{k}>0$ are the spinors of the (unique, if $\rho_{k} \neq \rho_{\ell} \forall k \neq l$ ) minimal ensemble that realizes $\boldsymbol{\rho}$, which therefore describes a pure state if and only if it has rank 1 as an operator.

Note that by Eq. (4.2), the density operator transforms under unitary operations as

$$
\boldsymbol{\rho} \mapsto \overline{(\boldsymbol{U} \circ \boldsymbol{\Psi}) \boldsymbol{E}_{+}(\boldsymbol{U} \circ \boldsymbol{\Psi})^{\sim}}=\boldsymbol{U} \overline{\boldsymbol{\Psi} \boldsymbol{E}_{+} \tilde{\boldsymbol{\Psi}}} \boldsymbol{U}^{\dagger}=\boldsymbol{U} \boldsymbol{\rho} \boldsymbol{U}^{\dagger} .
$$

Similarly, the ensemble-average expectation value of any observable $\boldsymbol{O}=\boldsymbol{O}^{\dagger} \in$ $\mathcal{G}_{3}^{\otimes N} / \boldsymbol{C}$ is

$$
\overline{\langle\psi|\underline{\boldsymbol{O}}| \psi\rangle} \leftrightarrow 2^{N} \overline{\left\langle\boldsymbol{E}_{+} \tilde{\boldsymbol{\Psi}} \boldsymbol{O} \boldsymbol{\Psi} \boldsymbol{E}_{+}\right\rangle_{0}}=2^{N}\left\langle\boldsymbol{O} \overline{\boldsymbol{\Psi} \boldsymbol{E}_{+} \tilde{\boldsymbol{\Psi}}}\right\rangle_{0} \equiv 2^{N}\langle\boldsymbol{O} \boldsymbol{\rho}\rangle_{0},
$$

just as shown in Eq. (2.17) for single qubit ensembles. In contrast to the case of a single qubit, however, the geometric interpretation of these observables is not straightforward. While one can certainly express $\boldsymbol{\rho}$ as a finite ensemble average $\sum_{k} p_{k} \boldsymbol{\Psi}_{k} \boldsymbol{E}_{+} \tilde{\boldsymbol{\Psi}}_{k}$ (where the $p_{k}>0$ are probabilities with $\sum_{k} p_{k}=1$ ), this decomposition is highly nonunique. The minimal ensemble obtained by diagonalization, on the other hand, will generally include entangled spinors $\boldsymbol{r}_{k}$, for which the expectation value $\left\langle\boldsymbol{O} \boldsymbol{r}_{k} \boldsymbol{r}_{k}^{\dagger}\right\rangle_{0}$ cannot be expressed as a product of inner products of the factors of $\boldsymbol{O}=\boldsymbol{O}^{1} \cdots \boldsymbol{O}^{N}$ with the polarization vectors of the individual qubits (indeed, $\boldsymbol{O}$ itself need not be factorizable!).

The best one can do is to expand $\rho$ in the product operator basis consisting of all $2^{2 N}$ products of the basis vectors $\boldsymbol{\sigma}_{\mu}^{q}$, i.e.

$$
\boldsymbol{\rho}=\sum_{\mu^{1}, \ldots, \mu^{N} \in\{0, x, y, z\}} \rho_{\mu^{1} \ldots \mu^{N}} \boldsymbol{\sigma}_{\mu^{1}}^{1} \cdots \boldsymbol{\sigma}_{\mu^{N}}^{N},
$$

where $\rho_{\mu^{1} \ldots \mu^{N}} \in \mathbb{R}$ and $\boldsymbol{\sigma}_{0}^{q} \equiv 1$ for notational convenience. The utility of this basis is most simply demonstrated via a concrete example, namely NMR spectroscopy. Here 
one is given a liquid sample consisting of identical molecules whose nuclear spins are chemically distinguishable, and hence constitutes an ensemble of multi-qubit systems (see [CPH98, HCST00, HSTC00] and references therein). The energy of interaction between the spins and an external magnetic field along $\mathrm{z}$ is given by an observable called the Zeeman Hamiltonian, $\boldsymbol{Z} \equiv\left(\omega^{1} \boldsymbol{\sigma}_{\mathrm{z}}^{1}+\cdots+\omega^{N} \boldsymbol{\sigma}_{\mathrm{z}}^{N}\right) / 2$, where $\omega^{q}$ is the energy difference between the $|0\rangle$ and $|1\rangle$ states of the $q$-th spin in the field. In thermal equilibrium at room temperatures, the polarization of the spins relative to the strongest available fields is typically $\alpha \sim 10^{-6}$, and the density operator of the ensemble is essentially $\boldsymbol{\rho}_{\text {eq }}=2^{-N}\left(1+\alpha\left(\boldsymbol{\sigma}_{\mathrm{z}}^{1}+\cdots+\boldsymbol{\sigma}_{\mathrm{z}}^{N}\right)\right)$. Via a suitable pulse of radio-frequency radiation, this may be rotated to $\boldsymbol{\rho}_{\mathrm{eq}}^{\prime} \equiv 2^{-N}\left(1+\alpha\left(\boldsymbol{\sigma}_{\mathrm{x}}^{1}+\cdots+\boldsymbol{\sigma}_{\mathrm{x}}^{N}\right)\right)$, which evolves under the interaction with the field as

$$
\begin{aligned}
e^{-\imath \boldsymbol{Z} t} \boldsymbol{\rho}_{\mathrm{eq}}^{\prime} e^{\boldsymbol{\iota} \boldsymbol{Z} t}= & 2^{-N}\left(1+\alpha\left(\cos \left(\omega^{1} t\right) \boldsymbol{\sigma}_{\mathrm{x}}^{1}-\sin \left(\omega^{1} t\right) \boldsymbol{\sigma}_{\mathrm{y}}^{1}+\cdots\right.\right. \\
& \left.\left.\cdots+\cos \left(\omega^{N} t\right) \boldsymbol{\sigma}_{\mathrm{x}}^{N}-\sin \left(\omega^{N} t\right) \boldsymbol{\sigma}_{\mathrm{y}}^{N}\right)\right) .
\end{aligned}
$$

Thus on measuring the total magnetization $M_{\mathrm{x}}$ along the $\mathrm{x}$ axis, $\boldsymbol{O} \equiv \gamma\left(\boldsymbol{\sigma}_{\mathrm{x}}^{1}+\right.$ $\cdots+\sigma_{\mathrm{x}}^{N}$ ) (where $\gamma$ is the nuclear gyromagnetic ratio), one obtains the sum of the projections of the rotating magnetization vectors of the spins along the $x$-axis, i.e.

$$
M_{\times}(t)=\alpha \gamma\left(\cos \left(\omega^{1} t\right)+\cdots+\cos \left(\omega^{N} t\right)\right),
$$

whose Fourier transform reveals the contribution from each spin. The way in which the factors of product operators transform like vectors under rotations accounts in large part for the computational utility of the product operator basis. Of course, unless it is a natural part of the problem at hand (as in NMR), one is better off not chosing a basis at all!

A stochastic quantum operation is a linear transformation of the density operator that may be written in operator sum form as [Kra83]

$$
\boldsymbol{\rho} \mapsto \Omega(\boldsymbol{\rho}) \equiv \sum_{k} \boldsymbol{Q}_{k} \boldsymbol{\rho} \boldsymbol{Q}_{k}^{\dagger},
$$

where the Kraus operators $\boldsymbol{Q}_{k} \in \mathcal{G}_{3}^{\otimes N} / \boldsymbol{C}$ satisfy $\sum_{k} \boldsymbol{Q}_{k}^{\dagger} \boldsymbol{Q}_{k}=1$. The term "stochastic" here ${ }^{3}$ refers to the fact that such an operation preserves the scalar part of $\rho$, since

$$
\langle\Omega(\boldsymbol{\rho})\rangle_{0}=\sum_{k}\left\langle\boldsymbol{Q}_{k} \boldsymbol{\rho} \boldsymbol{Q}_{k}^{\dagger}\right\rangle_{0}=\left\langle\boldsymbol{\rho} \sum_{k} \boldsymbol{Q}_{k}^{\dagger} \boldsymbol{Q}_{k}\right\rangle_{0}=2^{-N} .
$$

It is also easily seen that such quantum operations are positive, in that they preserve the positive-definiteness of $\boldsymbol{\rho}$; in fact, these operations have a yet stronger property known as complete positivity, meaning that if the qubits to which $\Omega$ applies are embedded in a larger system, then applying $\Omega$ to just those qubits preserves the positive-definiteness of the larger system's density operator. That this is a nontrivial extension of positivity is shown by the two-qubit "partial transpose" operator $\Omega_{\mathrm{T}}^{1}$, which carries $\sigma_{\mathrm{y}}^{1} \mapsto-\sigma_{\mathrm{y}}^{1}$ but leaves all the other operator factors unchanged; this is clearly positive on density operators not involving the second qubit, but acts on the density operator of the singlet state (Eq. (4.11)) as

$$
\begin{aligned}
& \boldsymbol{\psi}_{-} \equiv \frac{1}{4}\left(\sigma_{\mathrm{x}}^{1}+\sigma_{\mathrm{y}}^{1}-\sigma_{\mathrm{x}}^{2}-\sigma_{\mathrm{y}}^{2}\right) \boldsymbol{E}_{+}^{1} \boldsymbol{E}_{+}^{2}\left(\boldsymbol{\sigma}_{\mathrm{x}}^{1}+\sigma_{\mathrm{y}}^{1}-\sigma_{\mathrm{x}}^{2}-\sigma_{\mathrm{y}}^{2}\right) \\
&=\frac{1}{4}\left(1-\sigma_{\mathrm{x}}^{1} \sigma_{\mathrm{x}}^{2}-\sigma_{\mathrm{y}}^{1} \sigma_{\mathrm{y}}^{2}-\sigma_{\mathrm{z}}^{1} \sigma_{\mathrm{z}}^{2}\right) \\
& \mapsto \Omega_{\mathrm{T}}^{1}\left(\psi_{-}\right)=\frac{1}{4}\left(1-\sigma_{\mathrm{x}}^{1} \sigma_{\mathrm{x}}^{2}+\sigma_{\mathrm{y}}^{1} \sigma_{\mathrm{y}}^{2}-\sigma_{\mathrm{z}}^{1} \sigma_{\mathrm{z}}^{2}\right),
\end{aligned}
$$

\footnotetext{
${ }^{3}$ We prefer to avoid the more common but clumsy and matrix-bound term "trace-preserving".
} 
which has eigenvalues $[1 / 2,1 / 2,1 / 2,-1 / 2]$.

A quantum operation $\Omega$ is called unital if it preserves the identity itself, i.e. $\Omega(1)=1$, or equivalently, $\sum_{k} \boldsymbol{Q}_{k} \boldsymbol{Q}_{k}^{\dagger}=1$. Perhaps the most important example of a stochastic unital operation is found in the contraction ${ }^{4}$ by a single qubit $q \in$ $\{1, \ldots, N\}$, which may be written in operator sum form as [SCH98]:

$$
2\langle\boldsymbol{\rho}\rangle^{q} \equiv \boldsymbol{E}_{+}^{q} \boldsymbol{\rho} \boldsymbol{E}_{+}^{q}+\boldsymbol{E}_{-}^{q} \boldsymbol{\rho} \boldsymbol{E}_{-}^{q}+\boldsymbol{\sigma}_{\times}^{q}\left(\boldsymbol{E}_{+}^{q} \boldsymbol{\rho} \boldsymbol{E}_{+}^{q}+\boldsymbol{E}_{-}^{q} \boldsymbol{\rho} \boldsymbol{E}_{-}^{q}\right) \boldsymbol{\sigma}_{\times}^{q}
$$

This may also be expressed by dropping all terms in the product operator expansion of $\boldsymbol{\rho}$ depending on $q$, and multiplying the remaining terms by a factor of 2 . Note that, while $\left\langle_{-}\right\rangle^{q}$ is stochastic and unital, this factor means that the contraction itself is neither. The factor is nevertheless required if the result is to be interpreted as a density operator for the remaining qubits, since the contraction by the second qubit of the above singlet state is $\left\langle\boldsymbol{\psi}_{-}\right\rangle^{2}=1 / 4($ not $1 / 2)$.

This example also illustrates an important way in which general quantum operations are realized in practice, despite the fact that the universe as a whole evolves unitarily. As shown previously, the superposition state with spinor $\boldsymbol{\Psi}^{1}=$ $\left(1-\iota \sigma_{y}^{1}\right) / \sqrt{2}$ is converted into the singlet state with density operator $\boldsymbol{\psi}_{-}$by letting it interact with a second qubit so as to effect the c-NOT operation $N^{2 \mid 1}$. The contraction then corresponds to "discarding" the second qubit (i.e. ensuring that it does not further interact with the first and hence can be ignored), which yields the density operator $1 / 2$ of the totally mixed state for the first qubit. Since the basis states $\boldsymbol{E}_{ \pm}^{1}$ are unaffected by $\boldsymbol{N}^{2 \mid 1}$, the net quantum operation on the first qubit corresponds to what is known in quantum communications theory as the phase damping channel

$$
\boldsymbol{\rho} \mapsto(1-p) \boldsymbol{\rho}+p \boldsymbol{E}_{+} \boldsymbol{\rho} \boldsymbol{E}_{+}+p \boldsymbol{E}_{-} \boldsymbol{\rho} \boldsymbol{E}_{-}
$$

with damping parameter $p=1$. Phase damping is also known as $T_{2}$ relaxation in NMR, and as decoherence in quantum information processing; it is widely believed to be the dominant mechanism by which classical statistical mechanics arises from the underlying unitary dynamics $\left[\mathbf{G J K}{ }^{+} \mathbf{9 6}\right]$.

To illustrate the utility of geometric algebra in the study of general quantum operations, an eigenvalue characterization of stochastic, unital, one-bit quantum operations $\Omega$ will now be derived. This characterization was originally given by Fujiwara \& Algoet [FA99], although the derivation here parallels that more recently obtained using matrix methods King \& Ruskai [KR00]. This derivation will regard the Kraus operators $\boldsymbol{Q}_{k} \in \mathcal{G}_{3}$ as "complex quaternions" $\boldsymbol{A}_{k}+\boldsymbol{\iota} \boldsymbol{B}_{k}$ with $\boldsymbol{A}_{k}, \boldsymbol{B}_{k} \in$ $\mathcal{G}_{3}^{+}$, and consider the action of an arbitrary operation $\Omega$ on the scalar and vector parts of $\boldsymbol{\rho} \equiv(1+\boldsymbol{r}) / 2$ separately.

First, the action on 1 is

$$
\begin{aligned}
\Omega(1) & =\sum_{k}\left(\boldsymbol{A}_{k}+\iota \boldsymbol{B}_{k}\right)\left(\boldsymbol{A}_{k}+\iota \boldsymbol{B}_{k}\right)^{\dagger} \\
& \left.=\sum_{k}\left(\boldsymbol{A}_{k} \tilde{\boldsymbol{A}}_{k}+\boldsymbol{B}_{k} \tilde{\boldsymbol{B}}_{k}\right)+\iota \sum_{k}\left(\boldsymbol{B}_{k} \tilde{\boldsymbol{A}}_{k}-\boldsymbol{A}_{k} \tilde{\boldsymbol{B}}_{k}\right)\right) .
\end{aligned}
$$

The first summation is symmetric with respect to spatial reversion and inversion, i.e. scalar, while the second (excluding the $\iota$ ) is reversion antisymmetric but inversion symmetric, i.e. a bivector. Writing $\boldsymbol{A} \equiv \alpha+\boldsymbol{\iota} \boldsymbol{a}$ and $\boldsymbol{B} \equiv \beta+\boldsymbol{\iota} \boldsymbol{b}$, so that

\footnotetext{
${ }^{4}$ Otherwise known as the "partial trace".
} 
$\boldsymbol{A}_{k} \tilde{\boldsymbol{A}}_{k}+\boldsymbol{B}_{k} \tilde{\boldsymbol{B}}_{k}=\alpha_{k}^{2}+\left\|\boldsymbol{a}_{k}\right\|^{2}+\beta_{k}^{2}+\left\|\boldsymbol{b}_{k}\right\|^{2}$, we may further expand the second summation as follows:

$$
\begin{aligned}
& \sum_{k}\left[\left(\beta_{k}+\iota \boldsymbol{b}_{k}\right)\left(\alpha_{k}-\iota \boldsymbol{a}_{k}\right)-\left(\alpha_{k}+\iota \boldsymbol{a}_{k}\right)\left(\beta_{k}-\iota \boldsymbol{b}_{k}\right)\right] \\
&=\sum_{k}\left[\left(\alpha_{k} \beta_{k}+\iota\left(\alpha_{k} \boldsymbol{b}_{k}-\beta_{k} \boldsymbol{a}_{k}\right)+\boldsymbol{b}_{k} \boldsymbol{a}_{k}\right)-\right. \\
&\left.\left(\alpha_{k} \beta_{k}+\iota\left(\beta_{k} \boldsymbol{a}_{k}-\alpha_{k} \boldsymbol{b}_{k}\right)+\boldsymbol{a}_{k} \boldsymbol{b}_{k}\right)\right] \\
&=\sum_{k}\left[2 \boldsymbol{\iota}\left(\alpha_{k} \boldsymbol{b}_{k}-\beta_{k} \boldsymbol{a}_{k}\right)-2 \boldsymbol{a}_{k} \wedge \boldsymbol{b}_{k}\right]
\end{aligned}
$$

Thus $\Omega$ is unital if and only if

$$
\sum_{k}\left(\alpha_{k} \boldsymbol{b}_{k}-\beta_{k} \boldsymbol{a}_{k}\right)=\sum_{k} \boldsymbol{a}_{k} \times \boldsymbol{b}_{k}
$$

(where " $\times$ " denotes the cross product) and

$$
\sum_{k}\left(\alpha_{k}^{2}+\left\|\boldsymbol{a}_{k}\right\|^{2}+\beta_{k}^{2}+\left\|\boldsymbol{b}_{k}\right\|^{2}\right)=1 .
$$

Similarly, the action on $\boldsymbol{r} \in \mathfrak{R}_{3}$ is

$$
\begin{aligned}
\Omega(\boldsymbol{r}) & =\sum_{k}\left(\boldsymbol{A}_{k}+\iota \boldsymbol{B}_{k}\right) \boldsymbol{r}\left(\tilde{\boldsymbol{A}}_{k}-\iota \tilde{\boldsymbol{B}}_{k}\right) \\
& =\sum_{k}\left(\boldsymbol{A}_{k} \boldsymbol{r} \tilde{\boldsymbol{A}}_{k}+\boldsymbol{B}_{k} \boldsymbol{r} \tilde{\boldsymbol{B}}_{k}\right)+\iota \sum_{k}\left(\boldsymbol{B}_{k} \boldsymbol{r} \tilde{\boldsymbol{A}}_{k}-\boldsymbol{A}_{k} \boldsymbol{r} \tilde{\boldsymbol{B}}_{k}\right) .
\end{aligned}
$$

The first summation is over different dilation/rotations of $\boldsymbol{r}$; the second summation (excluding the $\iota$ ) is reversion and inversion antisymmetric, i.e. a trivector, and may be further expanded as above:

$$
\begin{aligned}
& \sum_{k}\left[\left(\beta_{k}+\iota \boldsymbol{b}_{k}\right) \boldsymbol{r}\left(\alpha_{k}-\iota \boldsymbol{a}_{k}\right)-\left(\alpha_{k}+\iota \boldsymbol{a}_{k}\right) \boldsymbol{r}\left(\beta_{k}-\iota \boldsymbol{b}_{k}\right)\right] \\
= & \sum_{k}\left[\left(\alpha_{k} \beta_{k} \boldsymbol{r}+\boldsymbol{\iota}\left(\alpha_{k} \boldsymbol{b}_{k} \boldsymbol{r}-\beta_{k} \boldsymbol{r} \boldsymbol{a}_{k}\right)+\boldsymbol{b}_{k} \boldsymbol{r} \boldsymbol{a}_{k}\right)-\right. \\
& \left.\left(\alpha_{k} \beta_{k} \boldsymbol{r}+\boldsymbol{\iota}\left(\beta_{k} \boldsymbol{a}_{k} \boldsymbol{r}-\alpha_{k} \boldsymbol{r} \boldsymbol{b}_{k}\right)+\boldsymbol{a}_{k} \boldsymbol{r} \boldsymbol{b}_{k}\right)\right] \\
= & \sum_{k}\left[2 \iota \alpha_{k} \boldsymbol{b}_{k} \cdot \boldsymbol{r}-2 \iota \beta_{k} \boldsymbol{a}_{k} \cdot \boldsymbol{r}+2 \boldsymbol{b}_{k} \wedge \boldsymbol{r} \wedge \boldsymbol{a}_{k}\right]
\end{aligned}
$$

Multiplying through by $-\iota / 2$ converts this to

$$
\sum_{k}\left[\alpha_{k} \boldsymbol{b}_{k} \cdot \boldsymbol{r}-\beta_{k} \boldsymbol{a}_{k} \cdot \boldsymbol{r}-\left(\boldsymbol{\iota}\left(\boldsymbol{a}_{k} \wedge \boldsymbol{b}_{k}\right)\right) \cdot \boldsymbol{r}\right],
$$

which vanishes if and only if

$$
\sum_{k}\left(\alpha_{k} \boldsymbol{b}_{k}-\beta_{k} \boldsymbol{a}_{k}\right) \cdot \boldsymbol{r}=\sum_{k}\left(\boldsymbol{b}_{k} \times \boldsymbol{a}_{k}\right) \cdot \boldsymbol{r} .
$$

If $\Omega$ is stochastic, this must be true for all $\boldsymbol{r}$, which is equivalent to

$$
\sum_{k}\left(\alpha_{k} \boldsymbol{b}_{k}-\beta_{k} \boldsymbol{a}_{k}\right)=\sum_{k} \boldsymbol{b}_{k} \times \boldsymbol{a}_{k} .
$$

A comparison with Eq. (4.26) shows further that $\Omega$ is both unital and stochastic if and only if $\sum_{k} \alpha_{k} \boldsymbol{b}_{k}=\sum_{k} \beta_{k} \boldsymbol{a}_{k}$ and $\sum_{k} \boldsymbol{a}_{k} \times \boldsymbol{b}_{k}=0$.

If we regard a stochastic $\Omega$ as an affine transformation, i.e.

$$
\Omega\left(\frac{1}{2}(1+\boldsymbol{r})\right)=\frac{1}{2}\left(1+\boldsymbol{t}+\sum_{\mu}\left(\boldsymbol{\sigma}_{\mu} \cdot \boldsymbol{r}\right) \boldsymbol{s}_{\mu}\right)
$$

$\left(\boldsymbol{s}_{\mu}, \boldsymbol{t} \in \mathfrak{R}_{3}\right)$, we see from the derivation leading up to (4.26) that

$$
\begin{aligned}
\boldsymbol{t} & =2 \sum_{k}\left(\alpha_{k} \boldsymbol{b}_{k}-\beta_{k} \boldsymbol{a}_{k}-\boldsymbol{a}_{k} \times \boldsymbol{b}_{k}\right) \\
& =4 \sum_{k}\left(\alpha_{k} \boldsymbol{b}_{k}-\beta_{k} \boldsymbol{a}_{k}\right)=4 \sum_{k} \boldsymbol{b}_{k} \times \boldsymbol{a}_{k} \quad(\text { by }(4.32)) .
\end{aligned}
$$

Similarly, the above vectors $\boldsymbol{s}_{\mu}=\left\langle\Omega\left(\boldsymbol{\sigma}_{\mu}\right)\right\rangle_{1}$ are

$$
\boldsymbol{s}_{\mu}=\sum_{k}\left(\boldsymbol{A}_{k} \boldsymbol{\sigma}_{\mu} \tilde{\boldsymbol{A}}_{k}+\boldsymbol{B}_{k} \boldsymbol{\sigma}_{\mu} \tilde{\boldsymbol{B}}_{k}\right) \quad(\mu \in\{\mathrm{x}, \mathrm{y}, \mathrm{z}\}),
$$


i.e. a sum of independent dilation/rotations of each basis vector.

It follows that stochastic and unital quantum operations $\Omega$ may be characterized by finding conditions for the linear map $\boldsymbol{r} \mapsto\langle\Omega(\boldsymbol{r})\rangle_{1}=\Omega(\boldsymbol{r})$ to be written as a sum of dilation/rotations of $\boldsymbol{r}$. To this end, we expand $\boldsymbol{A}_{k} \boldsymbol{r} \tilde{\boldsymbol{A}}_{k}$ as

$$
\begin{aligned}
\left(\alpha_{k}+\iota \boldsymbol{a}_{k}\right) \boldsymbol{r}\left(\alpha_{k}-\iota \boldsymbol{a}_{k}\right) & =\alpha_{k}^{2} \boldsymbol{r}+\alpha_{k} \iota\left(\boldsymbol{a}_{k} \boldsymbol{r}-\boldsymbol{r} \boldsymbol{a}_{k}\right)+\boldsymbol{a}_{k} \boldsymbol{r} \boldsymbol{a}_{k} \\
& =\alpha_{k}^{2} \boldsymbol{r}+2 \alpha_{k} \boldsymbol{r} \times \boldsymbol{a}_{k}+2\left(\boldsymbol{r} \cdot \boldsymbol{a}_{k}\right) \boldsymbol{a}_{k}-\left\|\boldsymbol{a}_{k}\right\|^{2} \boldsymbol{r},
\end{aligned}
$$

with a similar expansion for $\boldsymbol{B}_{k} \boldsymbol{r} \tilde{\boldsymbol{B}}_{k}$. Thus on assuming that $\Omega$ is diagonal, i.e. $\boldsymbol{s}_{\mu} \equiv$ $\lambda_{\mu} \sigma_{\mu}=\Omega\left(\sigma_{\mu}\right)$, we get

$$
\begin{aligned}
\lambda_{\mu} \boldsymbol{\sigma}_{\mu}= & \boldsymbol{\sigma}_{\mu} \sum_{k}\left(\alpha_{k}^{2}+\beta_{k}^{2}-\left\|\boldsymbol{a}_{k}\right\|^{2}-\left\|\boldsymbol{b}_{k}\right\|^{2}\right)+2 \boldsymbol{\sigma}_{\mu} \times \sum_{k}\left(\alpha_{k} \boldsymbol{a}_{k}+\beta_{k} \boldsymbol{b}_{k}\right) \\
& +2 \sum_{k}\left(\left(\boldsymbol{\sigma}_{\mu} \cdot \boldsymbol{a}_{k}\right) \boldsymbol{a}_{k}+\left(\boldsymbol{\sigma}_{\mu} \cdot \boldsymbol{b}_{k}\right) \boldsymbol{b}_{k}\right) .
\end{aligned}
$$

Dotting both sides by $\sigma_{\mu}$ now yields

$$
\begin{aligned}
\lambda_{\mu} & =\sum_{k}\left(\alpha_{k}^{2}+\beta_{k}^{2}-\left\|\boldsymbol{a}_{k}\right\|^{2}-\left\|\boldsymbol{b}_{k}\right\|^{2}+2\left(\boldsymbol{\sigma}_{\mu} \cdot \boldsymbol{a}_{k}\right)^{2}+2\left(\boldsymbol{\sigma}_{\mu} \cdot \boldsymbol{b}_{k}\right)^{2}\right) \\
& =1-2 \sum_{k}\left(\left\|\boldsymbol{a}_{k}\right\|^{2}+\left\|\boldsymbol{b}_{k}\right\|^{2}-\left(\boldsymbol{\sigma}_{\mu} \cdot \boldsymbol{a}_{k}\right)^{2}-\left(\boldsymbol{\sigma}_{\mu} \cdot \boldsymbol{b}_{k}\right)^{2}\right) \\
& =1+2 \sum_{k}\left(\left(\boldsymbol{\sigma}_{\mu} \wedge \boldsymbol{a}_{k}\right)^{2}+\left(\boldsymbol{\sigma}_{\mu} \wedge \boldsymbol{b}_{k}\right)^{2}\right)
\end{aligned}
$$

so we have simple expressions for the eigenvalues. Now consider the vector obtained from the first line of this equation, i.e. $\sum_{\mu \in\{x, y, z\}} \lambda_{\mu} \sigma_{\mu}$

$$
\begin{aligned}
& =\sum_{\mu} \boldsymbol{\sigma}_{\mu} \sum_{k}\left(\alpha_{k}^{2}+\beta_{k}^{2}-\left\|\boldsymbol{a}_{k}\right\|^{2}-\left\|\boldsymbol{b}_{k}\right\|^{2}+2\left(\boldsymbol{\sigma}_{\mu} \cdot \boldsymbol{a}_{k}\right)^{2}+2\left(\boldsymbol{\sigma}_{\mu} \cdot \boldsymbol{b}_{k}\right)^{2}\right) \\
& =\boldsymbol{p}_{0} \sum_{k}\left(\alpha_{k}^{2}+\beta_{k}^{2}\right)+\sum_{\mu} \boldsymbol{p}_{\mu} \sum_{k}\left(\left(\boldsymbol{\sigma}_{\mu} \cdot \boldsymbol{a}_{k}\right)^{2}+\left(\boldsymbol{\sigma}_{\mu} \cdot \boldsymbol{b}_{k}\right)^{2}\right),
\end{aligned}
$$

where

$$
\begin{array}{ll}
p_{0} \equiv \sigma_{\mathrm{x}}+\sigma_{\mathrm{y}}+\sigma_{\mathrm{z}}, & p_{\mathrm{x}} \equiv \sigma_{\mathrm{x}}-\sigma_{\mathrm{y}}-\sigma_{\mathrm{z}}, \\
p_{\mathrm{y}} \equiv-\sigma_{\mathrm{x}}+\sigma_{\mathrm{y}}-\sigma_{\mathrm{z}}, & p_{\mathrm{z}} \equiv-\sigma_{\mathrm{x}}-\sigma_{\mathrm{y}}+\sigma_{\mathrm{z}} .
\end{array}
$$

Since the coefficients of the $\boldsymbol{p}$ 's are nonnegative and sum to 1 by (4.27), this shows that the vector $\sum_{\mu} \lambda_{\mu} \boldsymbol{\sigma}_{\mu}$ lies within the tetrahedron $\left\langle\boldsymbol{p}_{0}, \boldsymbol{p}_{\mathrm{x}}, \boldsymbol{p}_{\mathrm{y}}, \boldsymbol{p}_{\mathrm{z}}\right\rangle$, which is the condition on the eigenvalues found by Fujiwara and Algoet [FA99] as well as by King and Ruskai [KR00].

It is also known that an arbitrary linear map $\Omega$ has an operator sum representation if and only if it is completely positive [Sch96], so the above can also be viewed as a characterization of complete positivity for stochastic and unital maps of a single qubit's density operator. Finally, it is worth stressing once again that, because of the isomorphisms which exist between the Pauli algebra and the even subalgebra of the Dirac algebra, every step of the above derivation carries with it a natural interpretation in space-time, and is in fact even easier to carry out when the full power of the Dirac algebra is used.

In conclusion, it is hoped that the forgoing has given the reader a taste of the new insights which geometric algebra can provide into quantum information processing - and an appetite for more!

\section{References}

[Alt86] S. L. Altmann, Rotations, Quaternions and Double Groups, Oxford Univ. Press, 1986.

[Alt89] S. L. Altmann, Hamilton, Rodrigues, and the quaternion scandal, Math. Mag. 62 (1989), 291-308.

[Bay96] W. E. Baylis (ed.), Clifford (Geometric) Algebras, with Applications in Physics, Mathematics, and Engineering, Birkhauser, Boston MA, 1996. 
$\left[\mathrm{BBC}^{+} 95\right]$ A. Barenco, C. H. Bennett, R. Cleve, D. P. DiVincenzo, N. Margolus, P. Shor, T. Sleator, J. A. Smolin, and H. Weinfurter, Elementary gates for quantum computation, Phys. Rev. A 52 (1995), 3457-3467.

[BBDH93] W. E. Baylis, J. Bonenfant, J. Derbyshire, and J. Huschilt, Light polarization: A geometric algebra approach, Am. J. Phys. 61 (1993), 534-545.

[BD00] C. H. Bennett and D. P. DiVincenzo, Quantum information and computation, Nature 404 (2000), 247-255.

[Blo46] F. Bloch, Nuclear induction, Phys. Rev. 70 (1946), 460-474.

[Bro99] M. Brooks (ed.), Quantum Computing and Communications, Springer-Verlag, London, U.K., 1999.

[CEMM98] R. Cleve, A. Ekert, C. Macchiavello, and M. Mosca, Quantum algorithms revisited, Proc. R. Soc. A 454 (1998), 339-354.

[Cli78] W. K. Clifford, Applications of Grassmann's extensive algebra, Am. J. Math. 1 (1878), 350-358; see also Mathematical Papers by W. K. Clifford, (R. Tucker, ed.), Chelsea Publ. Co., Bronx NY, 1968.

[CPH98] D. G. Cory, M. D. Price, and T. F. Havel, Nuclear magnetic resonance spectroscopy: An experimentally accessible paradigm for quantum computing, Physica D 120 (1998), $82-101$.

[DHSvA93] C. J. L. Doran, D. Hestenes, F. Sommen and N. Van Acker, Lie groups as spin groups, J. Math. Phys. 34 (1993), 3642-3669.

[DLG93] C. J. L. Doran, A. N. Lasenby, and S. F. Gull, States and operators in the spacetime algebra, Found. Phys. 23 (1993), 1239-1264.

$[D L G+96]$ C. J. L. Doran, A. N. Lasenby, S. F. Gull, S. S. Somaroo, and A. D. Challinor, Spacetime algebra and electron physics, in Advances in Imaging and Electron Physics (P. Hawkes, ed.), Academic Press, Englewood Cliffs, NJ, 1996, pp. 271-386.

[EJ96] A. Ekert and R. Jozsa, Quantum computation and Shor's factorizing algorithm, Rev. Mod. Phys. 68 (1996), 733-753.

[EJ98] A. Ekert and R. Jozsa, Quantum algorithms: Entanglement enhanced information processing, Phil. Trans. R. Soc. Lond. A 356 (1998), 1769-1782.

[EK95] A. Ekert and P. L. Knight, Entangled quantum systems and the Schmidt decomposition, Am. J. Phys. 63 (1995), 415-423.

[FA99] A. Fujiwara and P. Algoet, One-to-one parametrization of quantum channels, Phys. Rev. A 59 (1999), 3290-3295.

[FH81] F. A. M. Frescura and B. J. Hiley, Geometric interpretation of the Pauli spinor, Am. J. Phys. 49 (1981), 152-157.

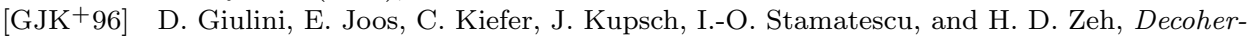
ence and the Appearance of a Classical World in Quantum Theory, Springer-Verlag, Berlin, FRG, 1996.

[HCST00] T. F. Havel, D. G. Cory, S. S. Somaroo, and C.-H. Tseng, Geometric algebra methods in quantum information processing by NMR spectroscopy, in Geometric Algebra with Applications in Science and Engineering (E. Bayro Corrochano and G. Sobczyk, eds.), Birkhauser, Boston, USA, 2001.

[HSTC00] T. F. Havel, S. S. Somaroo, C.-H. Tseng, and D. G. Cory, Principles and demonstrations of quantum information processing by NMR spectroscopy, Applicable Algebra in Engineering, Communications and Computing (T. Beth and M. Grassl, special edition eds.) 10 (2000), 339-374 (see also quant-ph/9812086).

[Hav01] T. F. Havel, Qubit logic, algebra and geometry, in Automated Deduction in Geometry (J. Richter-Gebert and D. Wang, eds.), Lect. Notes Artif. Intel., vol. 2061, SpringerVerlag, Berlin/Heidelberg, FRG, 2001.

[Hes66] D. Hestenes, Space-Time Algebra, Gordon and Breach, New York, NY, 1966.

[Hes99] D. Hestenes, New Foundations for Classical Mechanics (2nd ed.), Kluwer Academic Pub., Amsterdam, NL, 1999.

[HS84] D. Hestenes and G. Sobczyk, Clifford Algebra to Geometric Calculus, D. Reidel Pub. Co., Dordrecht, NL, 1984.

[Jan89] B. Jancewicz, Multivectors and Clifford Algebra in Electrodynamics, World Scientific Pub. Co., 1989.

[KR00] C. King and M. B. Ruskai, Minimal entropy of states emerging from noisy quantum channels, IEEE Trans. Info. Th. 47 (2001), 192-209 (see also quant/ph-9911079). 
[Kra83] K. Kraus, States, Effects and Operations, Springer-Verlag, Berlin, FRG, 1983.

[Llo95] S. Lloyd, Quantum-mechanical computers, Sci. Am. 273 (1995), 140-145.

[Per93] A. Peres, Quantum Theory: Concepts and Methods, Kluwer Academic Pub., Amsterdam, NL, 1993.

[Rie58] M. Riesz, Clifford Numbers and Spinors, Inst. for Fluid Dynamics \& Appl. Math., Univ. of Maryland, 1958; reprinted by Kluwer (eds. E. F. Bolinder \& P. Lounesto), Dordrecht, NL, 1993.

[Sak94] J. J. Sakurai, Modern Quantum Mechanics (revised ed.), Addison-Wesley Pub. Co., Reading, MA, 1994.

[Sch96] B. Schumacher, Sending entanglement through noisy quantum channels, Phys. Rev. A 54 (1996), 2614-2628.

[SCH98] S. S. Somaroo, D. G. Cory, and T. F. Havel, Expressing the operations of quantum computing in multiparticle geometric algebra, Phys. Lett. A 240 (1998), 1-7.

[SLD99] S. Somaroo, A. Lasenby, and C. Doran, Geometric algebra and the causal approach to multiparticle quantum mechanics, J. Math. Phys. 40 (1999), 3327-3340.

[Ste98] A. M. Steane, Quantum computing, Rep. Prog. Theor. Phys. 61 (1998), 117-173.

[Tol34] R. C. Tolman, Relativity, Thermodynamics and Cosmology, 1934; reprinted by Dover Publications, Inc., New York, NY, 1980.

[Tol38] R. C. Tolman, The Principles of Statistical Mechanics, 1938; reprinted by Dover Publications, Inc., New York, NY, 1980.

[vdW85] B. L. van der Waerden, A History of Algebra, Springer-Verlag, Berlin, FRG, 1985.

[Vla01] A. Y. Vlasov, Clifford algebras and universal sets of quantum gates, Phys. Rev. A 63 (1999), 054302.

[WC98] C. P. Williams and S. H. Clearwater, Ultimate Zero and One: Computing at the Quantum Frontier, Copernicus Books, Springer-Verlag, New York, NY, 1999.

T. F. Havel, BCMP, Harvard Medical School, Boston, MA, USA

Current address: NED, Massachusetts Institute of Technology, Cambridge, MA, USA

E-mail address, Timothy F. Havel: tfhavel@mit.edu

C. Doran, MraO, Cavendish laboratory, Madingley Road, Cambridge, CB3 0HE, UNITED Kingdom

E-mail address, Chris J. L. Doran: C.Doran@mrao.cam.ac.uk 